\title{
Synthesis and structure-activity relationships of teixobactin
}

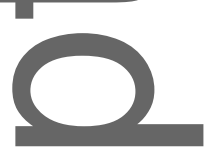

John A. Karas. ${ }^{1}$ Fan Chen, ${ }^{1}$ Elena K. Schneider-Futschik, ${ }^{1,2}$ Zhisen Kang, ${ }^{1}$ Maytham

Hussein, ${ }^{1}$ James Swarbrick, ${ }^{1}$ Daniel Hoyer, ${ }^{1,3,4}$ Andrew M. Giltrap, ${ }^{5}$ Richard J. Payne, ${ }^{5}$ Jian $\mathrm{Li}^{6}{ }^{6}$ and Tony Velkov ${ }^{1}$

'Department of Pharmacology \& Therapeutics, School of Biomedical Sciences, Faculty of Medicine, Dentistry and Health Sciences, the University of Melbourne, Parkville, Australia ${ }^{2}$ Lung Health Research Centre, Department of Pharmacology \& Therapeutics, the University of Melbourne, Parkville, Australia

${ }^{3}$ The Florey Institute of Neuroscience and Mental Health, the University of Melbourne,

Parkville, Australia

${ }^{4}$ Department of Molecular Medicine, the Scripps Research Institute, La Jolla, CA

${ }^{5}$ School of Chemistry, the University of Sydney, Sydney, Australia

${ }^{6}$ Monash Biomedicine Discovery Institute, Department of Microbiology, Monash University,

Clayton, Australia.

Address for correspondence: Tony Velkov and John Karas, Department of Pharmacology \& Therapeutics, School of Biomedical Sciences, Faculty of Medicine, Dentistry and Health Sciences, the University of Melbourne, Parkville, VIC 3010 Australia.

tony.velkov@unimelb.edu.au OR jkaras@unimelb.edu.au

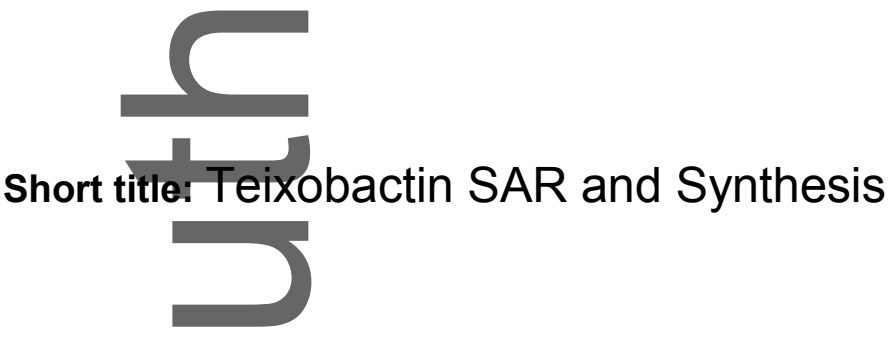

This is th auth $\mathrm{r}$ manuscript accepted for publication and has undergone full peer review but has not been throug - copyediting, typesetting, pagination and proofreading process, which may lead to differences between this version and the Version of Record. Please cite this article as doi:

10.1111/nyas.14282.

This article is protected by copyright. All rights reserved. 


\section{Graphical abstract:}

Our review provides a comprehensive treatise on the progress in understanding teixobactin (1)

chemistry, structure-activity relationships, and mechanisms of antibacterial activity.

Teixobactin represents an exciting starting point for the development of new antibiotics that can be used to combat multidrug-resistant bacterial ("superbug”) infections.

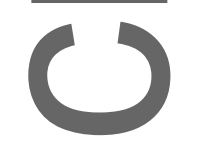

\section{Abstract}

The discovery of antibiotics has led to the effective treatment of bacterial infections that were otherwise fatal and has had a transformative effect on modern medicine. Teixobactin is an unusual depsipeptide natural product that was recently discovered from a previously unculturable a soil bacterium and found to possess potent antibacterial activity against several Gram positive pathogens, including methicillin-resistant Staphylococcus aureus and vancomycin-resistant Enterococci. One of the key features of teixobactin as an antibiotic lead is that resistance could not be generated in a laboratory setting. This is proposed to be a result of a mechanism of action that involves binding to essential cell wall synthesis building blocks, lipid II and lipid III. Since the initial isolation report in 2015, significant efforts have been made to understand its unique mechanism of action, to develop efficient synthetic routes for its production, and thus enable generation of analogues for structure-activity relationship studies and for optimization of its pharmacological properties. Our review provides a comprehensive treatise on the progress in understanding teixobactin chemistry, structure-activity-relationships, and mechanisms of antibacterial activity. Teixobactin represents an exciting starting point for the development of new antibiotics that can be used to combat multidrug-resistant bacterial ("superbug”) infections. 
Keywords: antimicrobial resistance; antimicrobial peptides; solid-phase peptide synthesis; teixobactin; structure-activity relationships; methicillin-resistant Staphylococcus aureus.

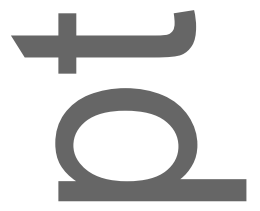

\section{Introduction}

Bad Bugs Need Drugs_-multidrug-resistant bacterial pathogens

Antimicrobial resistance $(A M R)$ is a serious global threat to human health and will inevitably lead to rising economic and social costs. The World Health Organisation (WHO) has warned of the possibility of a post-antibiotic era where common bacterial infections could become untreatable and the achievements of modern medicine undermined. ${ }^{1}$ The resultant health and financial implications suggest that global deaths could reach 10 million per year by 2050 with a cumulative cost to global economic output of $\$ 100$ trillion, far surpassing the burden of death by cancer. ${ }^{2}$ Notwithstanding the lack of investment from the pharmaceutical industry, several factors have contributed to the rising incidence of AMR. Antibiotic misuse in the agricultural sector has been a major cause, whereby antibiotics, such as colistin, are concomitantly used as a growth promoter in farm animals and therapeutically in humans. ${ }^{3}$

Recently, China has banned its use as a mitigation strategy. ${ }^{4}$ The widespread use of household antibacterial products with nonspecific actions, such as antiseptics and biocides, has also contributed to the rise in $\mathrm{AMR},{ }^{5}$ as has the overprescription and/or misuse of antibiotics in outpatient settings. ${ }^{6}$

Staphylococcus aureus isolates (methicillin-resistant, and vancomycin-intermediate and resistant) have been identified by the Infectious Diseases Society of America (IDSA) as among the top six most dangerous multidrug-resistant (MDR) microorganisms. ${ }^{7}$ Resistance of these Gram positive "superbugs"' to last-line antibiotics, such as vancomycin, has been increasing and is likely to far outpace the development of new drugs. ${ }^{8}$ Better stewardship of existing drugs is vital to delaying the widespread emergence of AMR. But there is still a 
pressing need for a new generation of antibiotics with novel mechanisms of action that are effective against resistant strains and that act on targets that are more resistant to mutation, i.e., "resistant to resistance." 9

Teixobactin-a new hope

Antimicrobial peptides produced via nonribosomal peptide (NRP) machinery have proven to be an invaluable pool of natural product scaffolds for the development of antimicrobial therapeutics. Teixobactin is a recently discovered antimicrobial peptide isolated from a previously uncultivable species of a soil bacterium called Eleftheria terrae (Fig. 1). ${ }^{10}$ An estimated $99 \%$ of all soil bacteria cannot be cultured via conventional laboratory techniques; ${ }^{11}$ therefore, a novel technology called the iChip was developed to overcome this challenge ${ }^{12}$ The device contains hundreds of miniature diffusion chambers that allow single microbes to be cultured in situ. It can produce monospecific cultures of previously uncultivable microbial organisms with higher yields than conventional methods. The technique thus enables access to secondary metabolites from previously untapped species. The power of technology is clearly demonstrated by the discovery of teixobactin, which represents a new class of antibiotic that displays a novel mechanism of action against Gram positive bacteria. ${ }^{10}$

The chemical structure of teixobactin can be described as a depsipeptide consisting of 11 amino acids with a characteristic cyclotetrapeptide macrocycle at the C-terminus and a methylated $\mathrm{N}$-terminus (Fig. 1). The structure contains five nonproteinogenic amino acids, including the rare L-allo-enduracididine (L-allo-End) and four D-amino acids: N-Me-D-Phe ${ }_{1}$, $\mathrm{D}-\mathrm{G} \mathrm{In}_{4}, \mathrm{D}-\mathrm{allo}-\mathrm{Ile}_{5}$, and D-Thr ${ }_{8}{ }^{13}$ Native teixobactin demonstrates narrow bactericidal activity against several Gram positive pathogens, such as S. aureus and Mycobacterium tuberculosis. ${ }^{10}$ It is also active against several MDR S. aureus strains, such as MRSA and VRE, which are associated with a wide range of infections in both community and 
nosocomial settings (e.g., bacteremia and pneumonia). ${ }^{10,14}$ Teixobactin is also a potent antimicrobial agent against Propionibacterium acnes that causes acne vulgaris and is the opportunistic pathogen in invasive infections. ${ }^{15}$ Teixobactin exhibited no cross-resistance with vancomycin and superior killing in late exponential phase populations. ${ }^{10,16}$ It is, however, ineffective against most species of Gram negative bacteria, presumably owing to an inability to cross the robust outer membrane of these organisms.

\section{Mechanism of action}

Teixobactin's unique mechanism of action is proposed to disrupt bacterial cell wall synthesis through binding and inhibition of the lipid II and lipid III substrates that are crucial for biosynthesis of the peptidoglycan structural component of the bacterial cell wall; thus leading to cell death via autolysis. ${ }^{10,17}$ The peptidoglycan component is crucial for maintaining structural integrity and thus cell survival and has served as a key target for several clinically approved antibiotics, including $\beta$-lactams and vancomycin. ${ }^{18}$ In Gram positive bacteria, the cell wall is primarily composed of peptidoglycan and cell wall teichoic acids (WTA). ${ }^{19}$ The peptidoglycan layer of Gram negative bacteria is generally much smaller than the Gram positive cell wall ( $\sim 1.5$ versus $\sim 20$ layers). Presumably, teixobactin is inactive against Gram negative bacteria as it cannot permeate the robust outer membrane, which is decorated with lipopolysaccharide (LPS). Initial studies with teixobactin failed to produce resistant mutants of $S$. aureus or M. tuberculosis; neither plating on media with low doses nor serial passaging over 27 days in the presence of subinhibitory levels of teixobactin led to detectable resistant mutants. ${ }^{10}$

Peptidoglycan is composed of linear chains of two alternating amino sugars, namely $\mathrm{N}$-acetylglucosamine (GlcNAc) and $\mathrm{N}$-acetylmuramic acid (MurNAc) that are covalently coupled, with the MurNAc subunit decorated with a pentapeptide chain (Fig. 2). The crosslinked matrix of these chains forms the backbone of the cell wall that allows the cell to 
elastically resist osmotic pressure. ${ }^{20}$ Synthesis of peptidoglycan begins with the formation of uridine diphosphate (UDP)-MurNAc functionalized with the pentapeptide, followed by coupling to an undecaprenyl pyrophosphate moiety, to form lipid I. UDP-GIcNAc is then coupled to lipid I to form lipid II, which is assembled on the cytosolic side of the bacterial membrane. Lipid II is then translocated across the periplasm where penicillin-binding proteins catalyze transglycosylation and transpeptidation reactions to incorporate the peptidoglycan subunits into the growing cell wall. This process releases undecaprenyl pyrophosphate, which is shuttled back to the cytosol where it is dephosphorylated and recycled. ${ }^{21}$ Lipid 11 becomes vulnerable to antimicrobial agents when it is localized on the outside of the bacterial inner membrane. It presents multiple binding sites for existing and potential antibiotio agents, such as the C-terminus of the pentapeptide domain, which is targeted by vancomycin ${ }^{22}$ or the pyrophosphate moiety that is targeted by lantibiotics and ramoplanin. ${ }^{23}$

Only a small quantity of lipid II exists in the cytoplasmic membrane at any given time ${ }^{18}$ which is indicative of a high turnover rate and/or rapid utilization for cell wall synthesis. As the rate-limiting step in peptidoglycan biosynthesis, inhibition of lipid II effectively cripples the integrity of the cell wall. ${ }^{21 a}$ Furthermore, the lipid II structure is unique within the bacterial cell kingdom, thereby preventing possible toxic effects of lipid II binders on mammalian cells. ${ }^{21 b}$ Available evidence suggests that teixobactin binds to the pyrophosphate moiety of lipid II but not to mature peptidoglycan that lacks pyrophosphate. ${ }^{21 a}$ This is possibly why it remains effective against vancomycin-resistant strains of bacteria and that no cross-resistance develops. ps. ${ }^{10}$

Cell wall teichoic acids (WTA) are present in roughly equal proportions with peptidoglycan in the Grampositive bacterial cell wall. ${ }^{19}$ Covalently attached to peptidoglycan units as polymers, they contribute to bacterial cell morphology and are implicated in some cases of antibiotic drug resistance. ${ }^{24}$ WTAs are composed of two components: a common linkage unit 
conserved across bacterial species that is covalently bound to MurNAc residues on peptidoglycan, and the main-chain polymer that consists of repeat units and that can vary across species (Fig. 2). Although there exists a complex and diverse array of possible repeat units, the most common ones consist of either glycerol-3-phosphate (such as those found in B. subtilis) or ribitol-5-phosphate (found in S. aureus). ${ }^{19,24}$ Accordingly, synthesis of WTAs is catalyzed by the Tag (teichoic acid glycerol) or Tar (teichoic acid ribitol) enzymes, depending on the type of polymer. This catalyzes the transfer of a GlcNAc residue onto a membranebound undecaprenyl-phosphate to form the intermediate lipid undecaprenyl-pyrophosphateGICNAc, ${ }^{25}$ also referred to as lipid III. ${ }^{10}$ It is purported that teixobactin also targets this key intermediate, which markedly reduces the supply of WTAs available for cell wall synthesis. ${ }^{17}$ The potential dual action of teixobactin targeting both lipid II and lipid III would have the potential to lead to a synergistic effect that increases cell wall lysis and bacterial killing compared with lipid II inhibition alone. ${ }^{17}$

\section{Chemical synthesis of teixobactin}

Given teixobaetin's enormous therapeutic potential, there is an incentive to further improve its effectiveness against Gram positive pathogens that are resistant to current drugs and potentially broaden its spectrum of activity. An important step towards this achievement is the development of a comprehensive understanding of its structure-activity relationships (SAR); this necessitates ready access to analogues and new and diverse chemical space. Therefore, the development of rapid and efficient chemical methods for producing teixobactin and its analogues is critical. For two recent reviews on teixobactin and analogues, we refer the reader to the two reports in Ref. 26.

There are two major challenges to consider when designing synthetic strategies for producing teixobactins, such as the formation of the C-terminal macrocycle (which possesses an ester bond) and the synthesis and incorporation of L-allo-End. For a 
comprehensive review on the synthesis of enduracididine analogues, see Brimble and coworkers. ${ }^{27}$ There are also several $\beta$-branched residues present, which can sometimes result in difficult couplings. Other nonstandard structural features include the four D-amino acids and an $\mathrm{N}$-methylated $\mathrm{N}$-terminus but these can be readily incorporated since the necessary building blocks are commercially available. Several strategies for producing teixobactin analogues already exist, such as a full stepwise strategy, convergent ligation, and macrocyclization on the solid support. The large-scale synthesis of the C-terminal macrocyclehas also been investigated as well as the evaluation of simplified analogues based on this scaffold. Each of these strategies is detailed below.

Total synthesis of teixobactin

The first report of a chemically synthesized teixobactin analogue utilized a stepwise 9-fluorenylmethyloxycarbonyl (Fmoc) solid-phase peptide synthesis (SPPS) approach (Fig. 3). ${ }^{28}$ After incorporation of residues 6-9 on the solid support, the semiprotected peptide was elongated via the D-Thr 8 side chain with L-lle ${ }_{11}$ and L-Arg 10 (as a replacement for L-allo-End) using $N^{\alpha}$ Alloe protection. The remaining $N$-terminal residues were then incorporated followed by acidolytic cleavage from the resin, cyclization in solution and global deprotection. The resultant compound maintained near-native activity against $S$. aureus (25923) and $B$. subtilis (6051) despite the Arg substitution at position 10, thus demonstrating that L-allo-End (which is synthetically difficult to access) is not critical for antimicrobial activity. This improves the economics for scale-up and enables rapid access to analogues for SAR studies .

Initially, two groups were able to overcome the difficulties associated with producing L-allo-End in useful quantities for the total synthesis of biologically active native teixobactin, ${ }^{29}$ to confirm that the original reported structure is correct. More recently, Rao and coworkers 
prepared a Fmoc SPPS-compatible L-allo-End precursor on a 30-gram scale, which led to a gram-scale synthesis of native teixobactin. ${ }^{30}$

Total synthesis of teixobactin using a linear approach

The first total synthesis to be reported was from Payne and coworkers. Here, the group adopted a stepwise Fmoc-SPPS strategy (Fig. 4), whereby L-Ser 7 and D-Thr 8 were incorporated at the C-terminus, followed by the formation of the ester bond between the $\beta$-hydroxyl group of threonine and Alloc-protected L-lle ${ }_{11}$. After chain elongation and Alloc deprotection, the final two residues were incorporated including synthetic bis-carboxybenzyl (Cbz)-protected L-allo-End ${ }_{10}$ (prepared in solution via seven synthetic steps). After mild acidic cleavage from the resin, the semiprotected peptide was subjected to lactamization to form the c-terminal macrocycle, followed by global deprotection with triflic acid to obtain native teixobactin ${ }^{29 a}$

Synthesis of analogues using a linear approach

Several other groups have adopted a similar linear approach to access analogues of the natural product. Specifically, in these studies, the full linear protected precursor is first assembled on a solid support, isolated via a cleavage step, cyclized, and then deprotected. Nowick et.al. assembled residues 1-10 with no side-chain protection on D-Thr ${ }_{8}$, followed by incorporation of $\mathrm{L}-1 \mathrm{e}_{11}$ via an esterification step (Fig. 5A). ${ }^{31}$ This approach was similar to that of Chan and coworkers, who also used unprotected D-Gln to $_{4}$ inimize steric bulk. ${ }^{32}$ Analogues generated by Brimble and coworkers differed to the previous examples in two ways: tert-butyldimethylsilyl (TBS) protection was used for the $\beta$-hydroxyl group of D-Thr 8 and the lactamization position was changed from between residues 10 and 11 to residues 8 and 9 (Fig. 5B) ${ }^{33}$ In another approach, Singh and coworkers performed the esterification directly after incorporation of $\mathrm{D}-\mathrm{Thr}_{8}$ and formed the macrolactamization between residues 9 and 10 (Fig. 5C). ${ }^{34}$ 
Total synthesis of teixobactin and analogues using a convergent approach

Li and coworkers took a very different approach to access native teixobactin by using a convergent serine-mediated ligation between the cyclic and linear fragments (Fig. 6A). ${ }^{35}$ The $\mathrm{N}$-terminal fragment bearing the reactive C-terminal salicylaldehyde ester was prepared using tert-butyloxycarbonyl (Boc)-SPPS and the C-terminal macrocyclic fragment was prepared by Fmoc-SPPS. ${ }^{29 b}$ A similar $6+5$ strategy was employed by Rao et al. to generate analogues of teixobactin (Fig. 6B), whereby semiprotected fragments were assembled and the conventional amide coupling performed. ${ }^{36}$ Yields of $\sim 5-15 \%$ were realized despite the possibility of epimerization of $\mathrm{L}^{-1 l \mathrm{e}_{6}}$ during $\mathrm{C}$-terminal activation of the $\mathrm{N}$-terminal fragment. Recently, the authors reported a $7+4$ strategy (with comparable yields), whereby a serinebased pseudo-proline moiety was incorporated at the $\mathrm{C}$-terminus of the $\mathrm{N}$-terminal fragment to suppress epimerization during the ligation (Fig. 6C). ${ }^{37}$ Each of these convergent strategies enables rapid access to teixobactin analogues since modifications can be incorporated into smaller peptide precursors that are easier to prepare, prior to the fragment condensation.

\section{On-resin cyclization}

One of the bottlenecks for rapid production of teixobactin analogues is the solution-phase macrocyclization step of the 8-11 segment, which often requires large reaction volumes to minimize oligomeric side products. To avoid this, Albericio and coworkers devised a strategy for producing $\operatorname{LyS}_{10}$ analogues whereby the $\varepsilon$-amino group was anchored to the solid support, thus enabling macrocyclization through judicious use of allyl-based protecting groups (Fig. 7A) ${ }^{38}$ After chain elongation of the $\mathrm{N}$-terminus, the unprotected peptide was then isolated from the resin and purified without further modification required. Recently, a more universal approach was described by Su et al. whereby L-Ala 9 was coupled to an aryl hydrazide linker that is compatible with Fmoc SPPS protocols. ${ }^{39}$ After chain assembly, the 
peptides were cyclized and concomitantly cleaved under mild oxidative conditions, followed by global deprotection with trifluoroacetic acid (Fig. 7B).

\section{Macrocycl}

There has been much interest in the unique 13-membered macrocyclic ring of teixobactin as this is important to enable access to chemically diverse analogues, yet this moiety has been identified as a potentially challenging structure to produce synthetically using solid-phase chemistry. Therefore, Reddy and coworkers attempted to optimize its synthesis via a solution-phase strategy. ${ }^{40}$ After assembly of the protected linear precursor with $\mathrm{Cbz}$ protection on L-allo-End 10 , a lanthanide triflate-mediated Shiina macrolactonization was performed. Unfortunately, Cbz removal from the guanidino-type moiety was incomplete during the final deprotection step, although a $\operatorname{Met}_{10}$ analogue was successfully produced via the same strategy (Fig. 8A). Jamieson et al. also used the C-terminal macrocycle as a scaffold to produce simplified analogues and incorporated cysteine at position 7 to enable a convenient chemoselective ligation between halogenated isoprenoid chains (farnesyl and geranyl) with the cyclic peptide intermediate bearing the $\beta$-thiol group (Fig. 8B). ${ }^{41}$ The Singh group have recently reported a novel approach for forming the macrocyclic ring, by replacing the ester linkage with a disulfide bond (Fig. 8C). ${ }^{42}$ This enables cyclization to proceed under ambient conditions and without the use of orthogonal protecting groups.

\section{Alternative approaches}

There have also been two other complementary approaches to the synthesis of teixobactin and related analogues. Xu and coworkers completed a synthesis of teixobactin that was performed all in solution (Fig. 9A). ${ }^{43}$ Recently, Rao's group reported a gram-scale synthesis of the native peptide, which was underpinned by efficient preparation of an L-allo-End precursor (Fig. 9B). ${ }^{30}$ This work represents a considerable synthetic effort and is highly significant since it could enable large-scale production of chemically diverse teixobactin 
analogues. In another approach, Zhang and coworkers utilized a chemoenzymatic approach to generate analogues. ${ }^{44}$ Specifically, linear peptides were first synthesized using standard SPPS and then cyclized by reaction with a purified biosynthetic thioesterase domain (Fig. 9C).

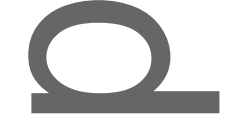

\section{Structure-activity relationships}

Several hundred teixobactin analogues have already been synthesized and evaluated since Lewis and coworkers' seminal report in $2015 .^{10}$ The general conclusions from the SAR studies are described below and comprehensively documented in the Supporting Information (online only). Refer also to Figure 10, which highlights the regions of teixobactin that are important for its antimicrobial activity. Table 1 also provides a concise summary of the modifications to date and identifies knowledge gaps relating to structure and activity.

\section{N-terminus}

Acetylation abolishes activity, indicating that a cationic $\mathrm{N}$-terminus is important ${ }^{34,45} \mathrm{~A}$ more comprehensive study also found that activity was lost upon acylation (e.g., decanoyl) and a bis-decanyl alkylation. ${ }^{38}$ This is consistent with the finding that the cationic $\mathrm{N}$-terminus is likely involved in binding of the pyrophosphate moiety of lipid II. ${ }^{46}$ However, dimethylation retained activity against $B$. subtilis. ${ }^{38-39}$ A mono-benzylated $\operatorname{Arg}_{10}$ analogue maintained some activity as did the nonmethylated $\mathrm{N}$-terminus.

\section{NMe-D-Phe 1}

Residue 1 is important for teixobactin's bactericidal effect, as Zong et al. demonstrated that its deletion abolished antimicrobial activity. ${ }^{36}$ This is consistent with a recent molecular dynamics study, which indicated that the aromatic ring interacts with the cell membrane surface. 47 Analogues bearing NMe-D-Ala 1 (i.e., those without the phenyl ring) were also found to be inactive ${ }^{48}$ (although activity is restored with the incorporation of L-Arg ${ }_{9}^{49}$ ), as was 
NMe-D-Lys ${ }_{1}{ }^{38}$ and other aromatic residues bearing heteroatoms. ${ }^{32,36,39}$ By contrast, replacement of the phenyl ring with various biphenyl derivatives enhances antimicrobial activity, ${ }^{30,36}$ which further supports the notion of side chain-membrane interactions. The $N^{\alpha}$ methyl group is not critical for activity; ${ }^{39,48 a, 50}$ replacement with a benzyl group is tolerated somewhat although not so for an $n$-decanyl group. ${ }^{50}$ Inversion of the stereochemistry at the a-carbon abolishes activity, which suggests that the altered orientation of the benzyl side chain could lead to interference in $\mathrm{N}$-terminal binding with the pyrophosphate group of lipid II. ${ }^{45,51}$ $L-I I e_{2}$ Alanine substitution of residue 2 renders teixobactin analogues inactive, ${ }^{48,52}$ which indicates its importance for its activity. Even substitution with structurally similar amino acids such as L-Leu and L-Val reduced potency somewhat. D-allo-lle, L-Ser, and L-Phe substitutions were also not tolerated, ${ }^{48 a}$ and unsurprisingly, nor was L-Lys. ${ }^{53}$ But once again, Parmar et al. were able to incorporate L-Ala 2 into L-Leu ${ }_{10}$-teixobactin and maintain antimicrobial activity, by inserting arginine at residues 3 and $4 .{ }^{49} \mathrm{~N}$-methylation was also found to abolish activity, ${ }^{37}$ possibly due to disruption of the supramolecular assembly of teixobactin and/or a conformational change that disrupts $\mathrm{N}$-terminal binding to the pyrophosphate group of lipid II. ${ }^{46}$ $\mathrm{L}-\mathrm{Ser}_{3}$ The $\beta$-hydroxyl group of residue 3 is not critical for teixobactin's bactericidal activity as several studies have shown that L-Ala substitution is well tolerated. ${ }^{48,52}$ Cationic replacement is also possible ${ }^{38,48 a, 49,53}$ but not so for O-phosphorylation ${ }^{36}$ or L-Asn incorporation. ${ }^{48 a} \mathrm{~N}$ methylation abolishes activity, ${ }^{37}$ presumably due to disruption of hydrogen bonding at the dimer interface between L-Ser 3 of one monomer and L-Ser ${ }_{7}$ of the other. ${ }^{46}$ 
$D-G / n_{4}$

Modification of the side chain of residue 4 leads mostly to analogues with moderate to good antimicrobial activity, such as those bearing D-Ala ${ }^{48 b, 52}$ or cationic residues. ${ }^{36,38-39,53}$ Several $\mathrm{Leu}_{10}$-teixobactins bearing D-Arg ${ }_{4}$ were found to be extremely potent. ${ }^{49}$ Stereochemical inversion abolishes activity, possibly due to steric clashes that prevent oligomerization..$^{45,48 a, 51}$ Replacement with D-Asn ${ }^{39,48 a}$ and dimethylaminoethyl-functionalized D-GIn ${ }^{36}$ led to analogues with relatively good activity. $N$-methylation reduced teixobactin's antimicrobial activity significantly without abolishing it completely ${ }^{37}$ it only attenuated oligomer assembly. This was used to good effect by Nowick's group as gelation was suppressed, which enabled crystallization and structural characterization of a Lys s $_{10}$ teixobactin analogue. ${ }^{46}$

D-allo-lle 5

Replacement with D-Ala abolishes activity ${ }^{48,52}$ unless other cationic substitutions are made elsewhere (vide supra). ${ }^{49} \mathrm{D}^{- \text {Lys }_{5}}$ rendered the analogue inactive ${ }^{53}$ and even a minor modification, such as D-Val substitution, reduced the antimicrobial killing effect. ${ }^{48 a}$ Inversion of the $\beta$-carbon stereochemistry is somewhat detrimental for teixobactin's bacterial killing; ${ }^{29 b}$ incorporation of L- $-\mathrm{ll}_{5}$ led to an inactive analogue. ${ }^{51}$ Activity was abolished after $\mathrm{N}$ methylation $L-I / e_{6}$

Three separate studies found that alanine substitution was not well tolerated, ${ }^{48,52}$ although Parmar et al. developed a potent analogue that also contained L-Arg at position $3 .{ }^{49}$ L-Lys was detrimental for antimicrobial activity, as were several hydrophobic substitutions (L-Leu, L-Phe, and L-Vall $)^{48 a}$ but an L-cyclohexylglycine (L-Chg) bearing analogue at position 6 did exhibit potency ${ }^{54} \mathrm{~N}$-methylation was tolerated somewhat, which indicates that the $\mathrm{N}-\mathrm{H}$ proton may not be involved in intermolecular hydrogen bonding. ${ }^{37}$ 
Deletion of the $\beta$-hydroxyl group is detrimental to activity, ${ }^{39,48 b, 52,54}$ but not when $D-G \ln _{4}$ is also mutated to D-Arg. ${ }^{49}$ Replacement with L-Lys also caused a loss of activity, although moderate activity against $B$. subtilis was retained. ${ }^{38,53} \mathrm{~N}$-methylation abolishes activity, ${ }^{37}$ possibly again due to disruption of intermolecular hydrogen bonding, according to the recent crystal structure reported by Nowick et al. ${ }^{46}$

$D-\mathrm{Thr}_{8}$

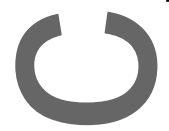

This residue is a key structural element in teixobactin, as its side chain is acylated by the $C$ terminus to form the pyrophosphate-binding macrocyclic motif. As expected, antimicrobial activity is abolished for linear analogues ${ }^{31}$ and when the stereochemistry is inverted. ${ }^{29 b, 31,51}$ Replacement of the lactone with a disulfide bond also results in redundant analogues ${ }^{42}$ but moderate activity is retained when a lactam is introduced via D-diaminopropionic acid (Dap).${ }^{54}$ When the more isosterically similar (2R,3S)-MeDap is incorporated, antimicrobial activity is improved, ${ }^{30,36}$ which could be owing to the enhanced stability of the amide bond compared with the ester bond. It also indicates the importance of the $\beta$-methyl group for antimicrobial potency.

L-Ala

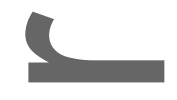

Substitution with L-Lys ${ }^{38,53-54}$ and L-Dap ${ }^{38}$ is tolerated somewhat. Jin et al. evaluated several analogues with variations at residue 9; stereochemical inversion and Gly substitution destroyed antimicrobial activity, most likely due to conformational changes in the macrocycle. L-Phe also led to inactive analogues but an L-Orn, analogue was found to have moderate activity. ${ }^{48 a}$ L-Arg is well tolerated ${ }^{49}$ as was aminobutyric acid, but dehydrobutyric acid less so. 32 
This amino acid presents a significant hurdle in the synthesis of teixobactin since it is not commercially available nor easily accessible synthetically. Indeed, in the first two reported total syntheses of native teixobactin, the L-allo-End analogue was prepared in 7 or 10 steps in $17 \%$ to $31 \%$ overall yield, respectively. ${ }^{29,55}$ Therefore, substitution of this residue has been a priority to enable rapid SAR studies for the development of potent analogues. Initial attempts at replacing the L-allo-End ${ }_{10}$ with L-Arg ${ }_{10}$, another guanidine-based amino acid, resulted in a product with a similar antibacterial spectrum profile as the native compound, albeit with slightly reduced efficacy against $S$. aureus and B. subtilis. ${ }^{28,34}$ Nevertheless, it demonstrated the feasibility of producing potent teixobactin analogues without the need for the precious L-allo-End ${ }_{10}$ amino acid. Several other analogues with substitutions at position 10 have also been evaluated. It was found that L-Orn 10 teixobactin ${ }^{29 b}$ possessed similar activity against Gram positive pathogens to the L-Arg ${ }_{10}$ analogue; L-Lys 10 had improved activity. ${ }^{31}$ Two independent studies evaluated a panel of compounds with residues isosterically similar to arginine at position 10 (such as citrulline and dimethylarginine); most were only moderately active but L-homo-Arg ${ }_{10}$ had promising activity. ${ }^{33,56}$ A separate study found that an L-Arg(Me) ${ }_{10}$, L-Nle ${ }_{11}$ teixobactin was potent and exhibited superior killing kinetics to vancomycin against $P$. acnes ATCC $11828 .^{32}$ Therefore, the prevailing logic was that a cationic residue at position 10 is critical for activity. However, this has been shown to not be the case as hydrophobic substitution, e.g., with leucine led to analogues that possessed identical potency as native teixobactin against VRE and MRSA isolates. ${ }^{49,52}$ This was confirmed by a separate study, whereby all natural L-amino acids and several substituted aromatic residues were incorporated at position $10 .^{57}$

$L-I I e_{11}$

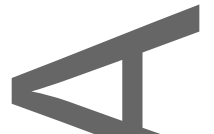

Alanine substitution is tolerated somewhat, with moderate ${ }^{48 b}$ to excellent ${ }^{49}$ antimicrobial activity reported for analogues with this variation. Lysine substitution is detrimental for 
activity, ${ }^{53}$ as is substitution with other hydrophobic residues, ${ }^{31,48 a}$ although L-Chg ${ }^{54}$ and L-Nle ${ }^{32}$ analogues were potent. An L-Cys ${ }_{11}$ analogue with side-chain cyclization was inactive as were all linear variants. ${ }^{42}$

SAR conclusions

In general, it was found that teixobactin is sensitive to alanine substitution at most positions;

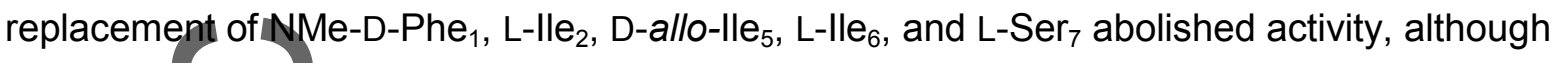
moderate activity was retained for D-Gln $\mathrm{n}_{4}$ L-allo-End ${ }_{10}$, and L-IIe ${ }_{11}$. Substitution of L-Ser 3 is tolerated, while L-Ala is already present at position 9 in the native natural product. Note that this general summary does not account for a separate study from Singh and coworkers, who found thatalanine substitution at positions $2,5,6$, and 7 is well tolerated for cationic teixobactin analogues. ${ }^{49}$ This knowledge helped inform the design of potent Leu/lle 10 analogues with arginine substitutions at positions 3,4 , and 9 , to mimic the net charge of the native peptide. ${ }^{42}$ The lysine scan has also been conducted; substitution of all hydrophobic residues abolished activity but was tolerated for polar noncharged residues. ${ }^{53} \mathrm{~A}$ similar study was performed whereby the net charge of teixobactin analogues was increased via incorporation of lysine and 2,3-diaminopropionic acid residues. It was found that the maximum net charge was +3-4 without abolishing activity. ${ }^{38}$ Inversion of stereochemistry has also been investigated but in general was found to lead to a loss in activity, ${ }^{45,48 a, 51}$ although analogues with D-Arg ${ }_{10}$ or D-allo-lle ${ }_{11}$ were somewhat tolerated. ${ }^{31}$

The unique, 13-membered C-terminal macrocycle of teixobactin is critical for activity as it is believed to bind to the pyrophosphate moiety of lipid II. Therefore, it is likely to be sensitive to any modifications that alter its active backbone conformation. Unsurprisingly, it has been shown that replacing D-Thr $r_{8}$ with its $\mathrm{L}$ isomer abolishes activity against several different Gram positive bacterial isolates. ${ }^{29 b, 31}$ By contrast, the replacement of D-Thr with D-Dap does not significantly alter activity (even without the $\beta$-methyl group) since the basic geometry of 
the macrocyclic backbone is largely maintained. ${ }^{54}$ Incorporating (2R,3S)-D-Dap further improves potency, indicating that the methyl group contributes to antimicrobial activity (vide supra). ${ }^{36}$ A separate study attempted to replace the depsipeptide ester bond with a disulfide bond to simplify the synthesis but this led to inactive analogues. ${ }^{42}$ Structure-minimized analogues have also been produced in an attempt to achieve this. In a recent study from Girt et al. the $6 \mathrm{~N}$-terminal residues were replaced with isoprenoids to promote interaction with the lipidic moiety of lipid II but only a farnesyl-bearing Lys $_{10}$ analogue possessed moderate activity. ${ }^{41}$ Similarly, a separate study that incorporated a lauroyl moiety in place of the first 5 residues retained low potency. ${ }^{54}$

The N-terminal tail of teixobactin is also essential for full antimicrobial activity. There is mounting evidence that antiparallel dimerization and further assembly into amphiphilic $\beta$-sheets are intrinsic to its mechanism of action. ${ }^{30,46,58}$ This is facilitated via hydrogen bonding along teixobactin's linear domain. The oligomerization hypothesis is supported by recent studies that demonstrated that $\mathrm{N}$-methylation of key residues, such as L-IIe ${ }_{2}$ and L$\mathrm{Ser}_{3}$, abolishes activity. ${ }^{30,37}$ The teixobactin dimer forms two cavities comprising the Cterminal macrocycle and the $\mathrm{N}$-terminus that acts as receptors for the pyrophosphate moiety via hydrogen bonding, ${ }^{30,46}$ which is most likely why N-terminal acylation or dialkylation is detrimental to activity. ${ }^{50}$ Incorporation of bis-aryl groups at position 1 can enhance activity, indicating a role in membrane insertion. ${ }^{30,36}$

\section{Structure-conformational relationships}

Several structure-based studies have been conducted on teixobactin to characterize its binding mode with lipid II and identify its active conformation. The Singh group probed the stereochemistry of all residues with the D-configuration and correlated antimicrobial activity with their NMR structures. ${ }^{51}$ Intriguingly, antimicrobial activity was abolished for all stereoisomers that possessed a highly ordered N-terminal tail (all except NMe-L-Phe ${ }_{1}$ ), 
which suggested that they possessed unfavorable conformations for dimerization and higher-order assembly. A crystal structure of a simplified analogue, whereby residues 1-5 were replaced with an acetyl group (which suppressed gelation and facilitated crystallization) indicated several important structural features and intramolecular interactions. ${ }^{54}$ It was found that the $N^{\alpha}$ protons of the C-terminal macrocycle were oriented in the same direction. Therefore, it was proposed that these amide hydrogens form hydrogen bonds with the pyrophosphate moiety of lipid II, which is a similar mode of binding as that of the food preservative nisin 59

Molecular dynamics studies have also been employed to aid in the elucidation of the interactions between teixobactin and lipid II. Liu and coworkers identified four predominant binding modes, showing further evidence of interaction between the pyrophosphate group and the C-terminal residues. ${ }^{60}$ The simulations also indicated that MurNAc may play a role in binding and suggested that teixobactin interacts with lipid II on a 2:1 basis, probably as a dimer. A separate study performed the simulations at a membrane surface and drew similar conclusions regarding pyrophosphate binding, but suggested that the side chains of L-Ser 7 and L-allo-End ${ }_{10}$ may also play a role. ${ }^{47} \mathrm{~A}$ separate finding suggested that the primary role of the hydrophobic residues was for membrane insertion. Öster et al. postulated that the Nterminal tail may play a greater functional role in the potent antimicrobial activity of teixobactin. ${ }^{58}$ On the basis of NMR chemical shift data, they deduced that the side chain of D-Gln ${ }_{4}$ may bind to D-Glu in the pentapeptide of lipid II. Furthermore, they indicated that the aggregation-prone $\mathrm{N}$-terminal region (when in the presence of lipid II) may be intrinsic to its activity. This notion was supported by X-ray crystallographic data, which found that a teixobactin analogue formed a supramolecular assembly, based on a $\beta$-sheet dimeric subunit. ${ }^{46}$ Therefore, it was proposed that these amyloid-like structures act as multivalent receptors for the pyrophosphate moiety of lipid II. We have recently constructed molecular 
models for these supramolecular assemblies (Fig. 11A) and proposed interactions of teixobactin with lipid II (Fig.11B).

\section{Toward the clinic}

Despite teixobactin's enormous therapeutic potential, there are still several hurdles that must be overcome for it to reach the clinic. Many anti-infective agents that are effective against MRSA already exist, including daptomycin and doxycycline. Therefore, teixobactin should display superior pharmacological properties to these drugs to justify an expensive and risky clinical trial program. ${ }^{61}$ The cost of goods is another important consideration, especially for analogues that require chemical synthesis, although progress is being made on this front. ${ }^{30}$ To enhance its clinical potential, several groups have attempted to broaden its spectrum of activity to enable the killing of problematic Gram negative pathogens. Bester and coworkers have found that cationic analogues possess a minor bactericidal effect against $P$. aeruginosa ATCC $27853 .{ }^{62} \mathrm{Ng}$ et al. combined teixobactin analogues with colistin, which is active against Gram negative bacteria. ${ }^{32}$ An improved bactericidal effect was observed, suggesting that the membrane-disrupting effect of colistin enables teixobactin access to its molecular target, lipid II. A similar approach was adopted by the Vederas group, whereby combination with polymyxin B nonapeptide and unacylated tridecaptin also elicited an antimicrobial effect against E. coli, S. enterica, and K. pneumoniae. ${ }^{63}$ These promising results could lead to safer therapeutic options for the treatment of recalcitrant Gram negative infections that currently require large doses of last-line antibiotics, such as colistin, which is nephrotoxic.

\section{Conclusions}

Teixobactin and its analogues are a recently discovered novel class of antibiotics that typically possess a narrow spectrum of activity against Gram positive bacteria. The most notable property of teixobactin is that it has not been possible to generate resistance against the molecule in the laboratory. There have been several strategies that have been adopted 
for the chemical synthesis of the natural product, which has led to the assembly and evaluation of several hundred analogues. This has underpinned numerous SAR studies that has led to the identification of key features of the teixobactin scaffold for antibacterial activity and binding to its target lipid II. Structural studies have revealed a potentially complex mode of action involving aggregate formation into macromolecular $\beta$-amyloid-like structures. Further development of this important field requires additional structure-conformation studies of target complexes, to delve deeper into the interactions of the teixobactin pharmacophore. This will ultimately lead to the development of more focused analogues through rationaldesign. Notwithstanding, the discovery of novel, highly potent broadspectrum teixobactin analogues holds immense potential for the development of therapeutics that are effective against drug-resistant bacterial strains and addresses the threat of apost-antibiotic world.

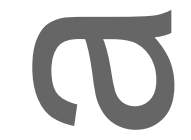

\section{Author contributions}

All authors have contributed to the writing of this manuscript and approved the final version.

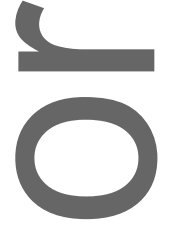

\section{Acknowledgements}

This work was funded by the Australian National Health and Medical Research Council (NHMRC, APP1128891). T.V. and J.L are also supported by a research Grant from the National Institute of Allergy and Infectious Diseases (NIAID) of the National Institutes of Health (RO1Al111965). The content is solely the responsibility of the authors and does not necessarily represent the official views of the National Institute of Allergy and Infectious 
Diseases of the National Institutes of Health. J. L. is an Australian National Health and Medical Research Council (NHMRC) Principal Research Fellow, and T. V. is an Australian NHMRC Industry Career Development Level 2 Research Fellow. E.K.S.-F. is an Australian NHMRC Peter Doherty Research Fellow.

\section{Competing interests:}

The authors declare no competing interests.

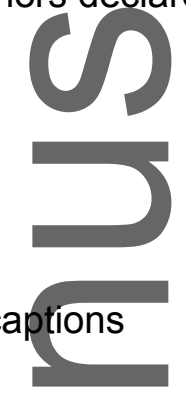

Figure 1. Structure of native teixobactin.

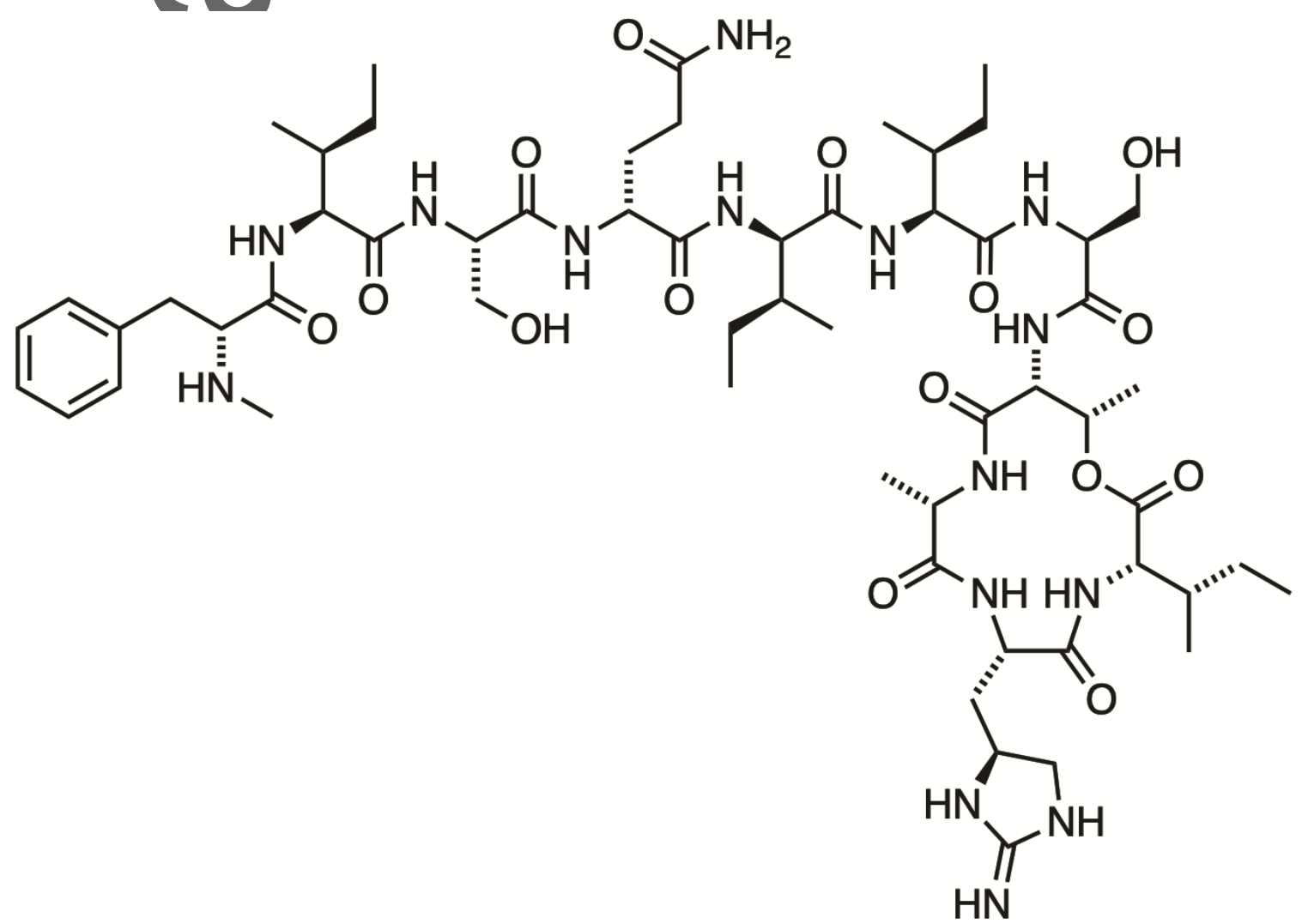

This article is protected by copyright. All rights reserved. 
Figure 2. The Gram positive bacterial cell wall is commonly composed of a dense peptidoglycan layer, which is further covalently modified with cell wall teichoic acid (WTA). Schematic diagram depicting the biosynthetic pathways for peptidoglycan and WTA from the common precursors $\mathrm{N}$-acetylglucosamine (NAG), N-acetylmuramic acid, (NAM), NAcetylmannosamine (ManAc) and undecaprenyl-pyrophosphate (Und-PP). Both the peptidoglycan (Lipid II) and WTA precursors are synthesized intracellularly using an Und-PP carrier lipid and then transported outside the cell where assembly into their respective biopolymers ensues. The Und-P carrier is then flipped back inside the cell and recycled in the production of more Lipidll and WTA precursors. Teixobactin inhibits peptidoglycan biosynthesis by binding to the pyrophosphate and the first sugar regions of lipid II, this results in autolysis and bacterial cell killing.

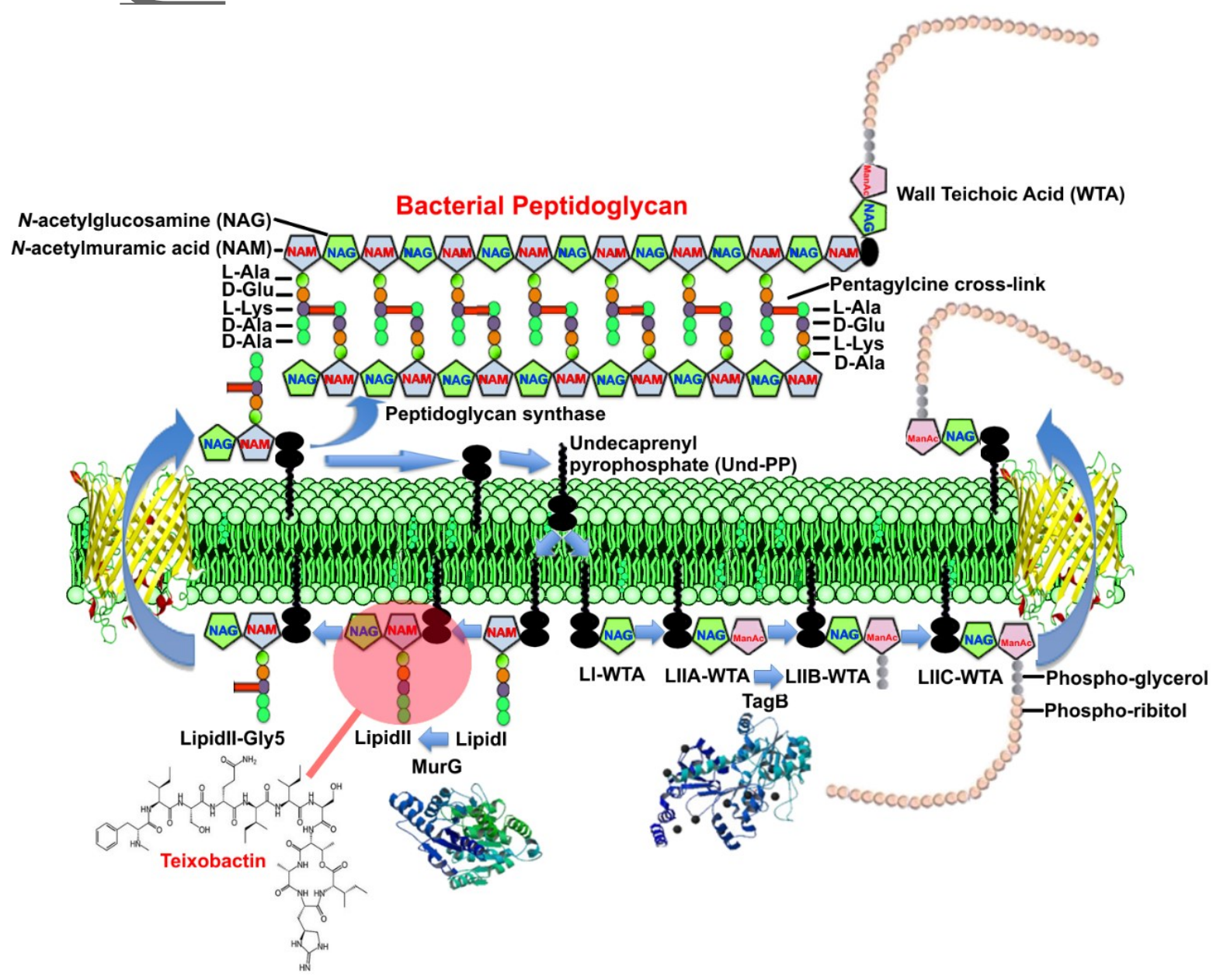

This article is protected by copyright. All rights reserved. 
Figure 3. Stepwise synthesis of $\mathrm{Arg}_{10}$ teixobactin by Albericio et al. (a) Alloc-Ile-OH, DIC, and DMAP; (b) Pd(PPh $)_{4}$ and $\mathrm{PhSiH}_{3}$; (c) Alloc-Arg(Pbf)-OH, HATU, and DIEA; (d) Fmoc SPPS; (e)Pd(PPh $)_{4}$ and PhSiH3; (f) 1\% TFA in DCM; (g) PyAOP, Oxyma Pure, and DIEA;

(h) TFA, TIS, and $\mathrm{H}_{2} \mathrm{O}$; (i) RP-HPLC. Yield $=6 \%$.

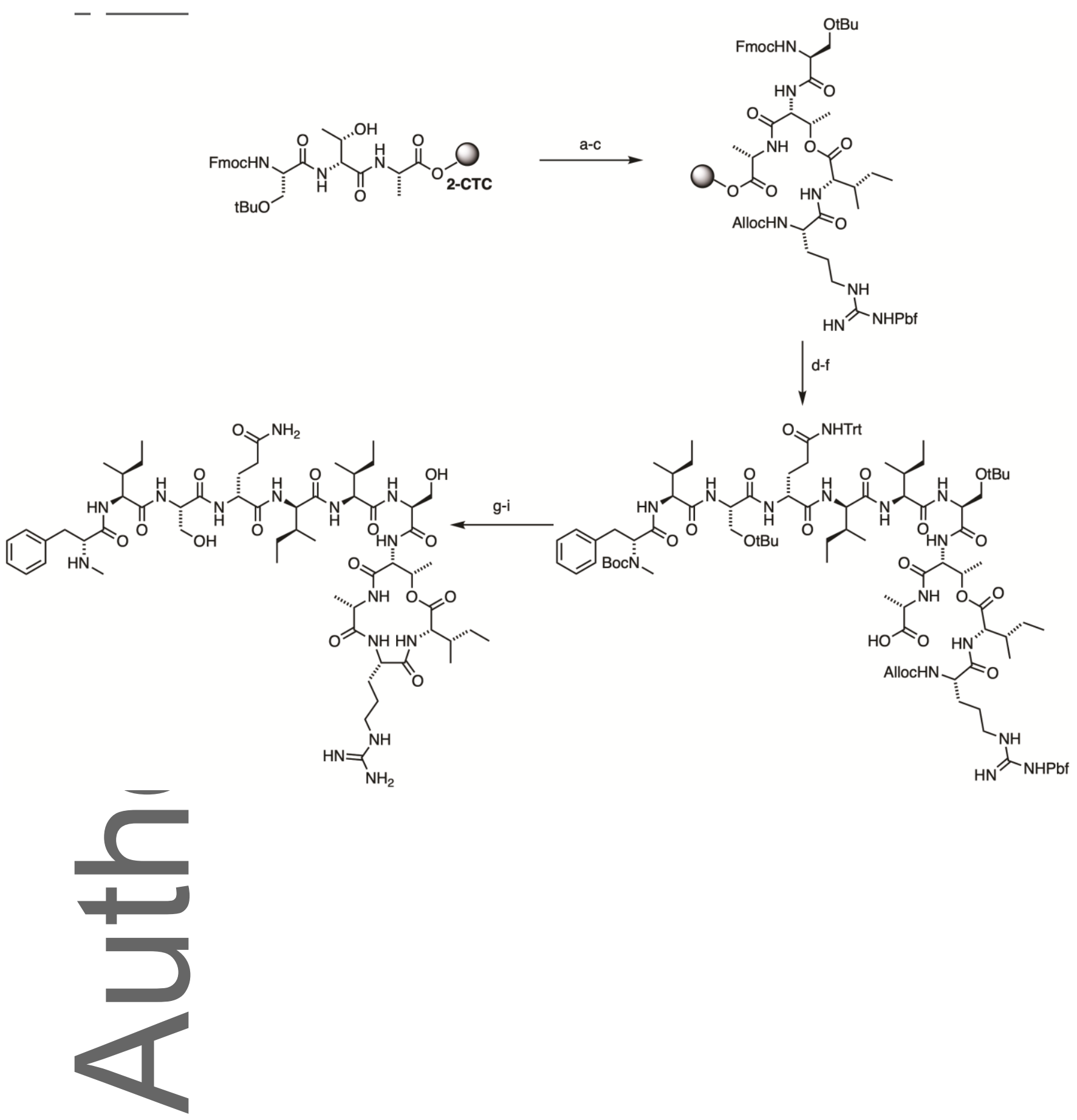

This article is protected by copyright. All rights reserved. 
Figure 4. Stepwise synthesis of native teixobactin by Payne et al. (a) Alloc-lle-OH, DIC, DMAP; (b) Fmoc SPPS; (c) Pd( $\left(\mathrm{PPh}_{3}\right)_{4}$ and PhSiH ${ }_{3}$; (d) Fmoc-End(Cbz) $)_{2}-\mathrm{OH}$, HATU, HOAt, and DIEA; (e) 10\% piperidine (30 s); (f) Fmoc-Ala-OH, PyBOP, and NMM; (g) 10\% piperidine; (h) 1\%TFA in DCM; (i) DMTMM.BF 4 and DIEA; (j) TFA, thioanisole, TfOH, and m-cresol; and (k) RP-HPLC. Yield $=3.3 \%$.

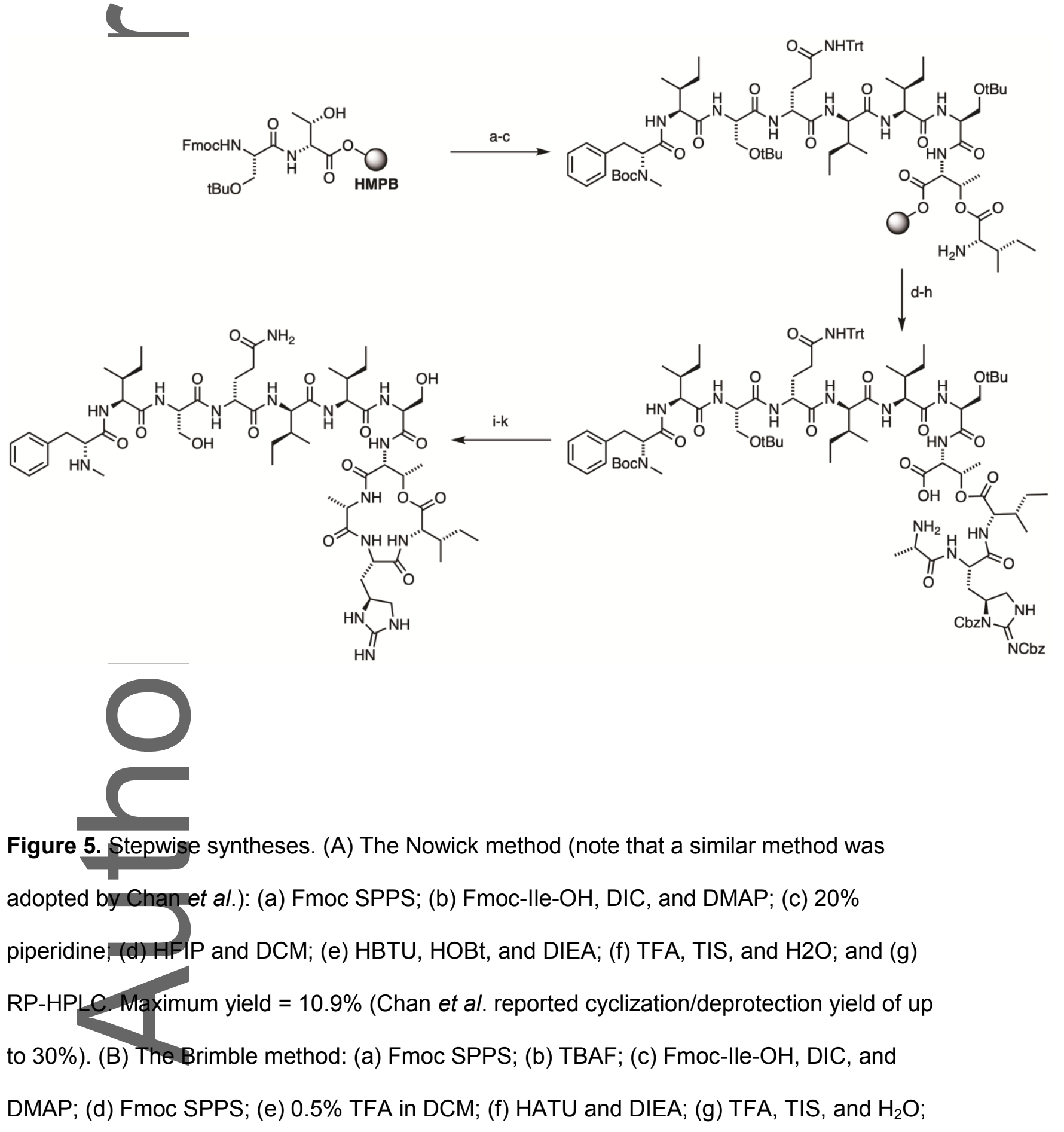

This article is protected by copyright. All rights reserved. 
and $(\mathrm{h})$ RP-HPLC. Maximum yield $=11.8 \%$. (C) The Singh method: (a) 20\% piperidine; (b) Alloc-D-Thr-OH, HATU, and DIEA; (c) Fmoc-lle-OH, DIC, and DMAP; (d) Fmoc SPPS; (e) Trt-CI, TEA; (f) Pd( $\left(\mathrm{PPh}_{3}\right)_{4}$, and $\mathrm{PhSiH}_{3}$; (g) Fmoc SPPS; (h) 2\% TFA, TIS in DCM; (i) HATU and DIEA; (j) TFA, TIS, and $\mathrm{H}_{2} \mathrm{O}$; and (k) RP-HPLC. The yield of $22 \%$ reported is presumably based on the crude linear peptide.

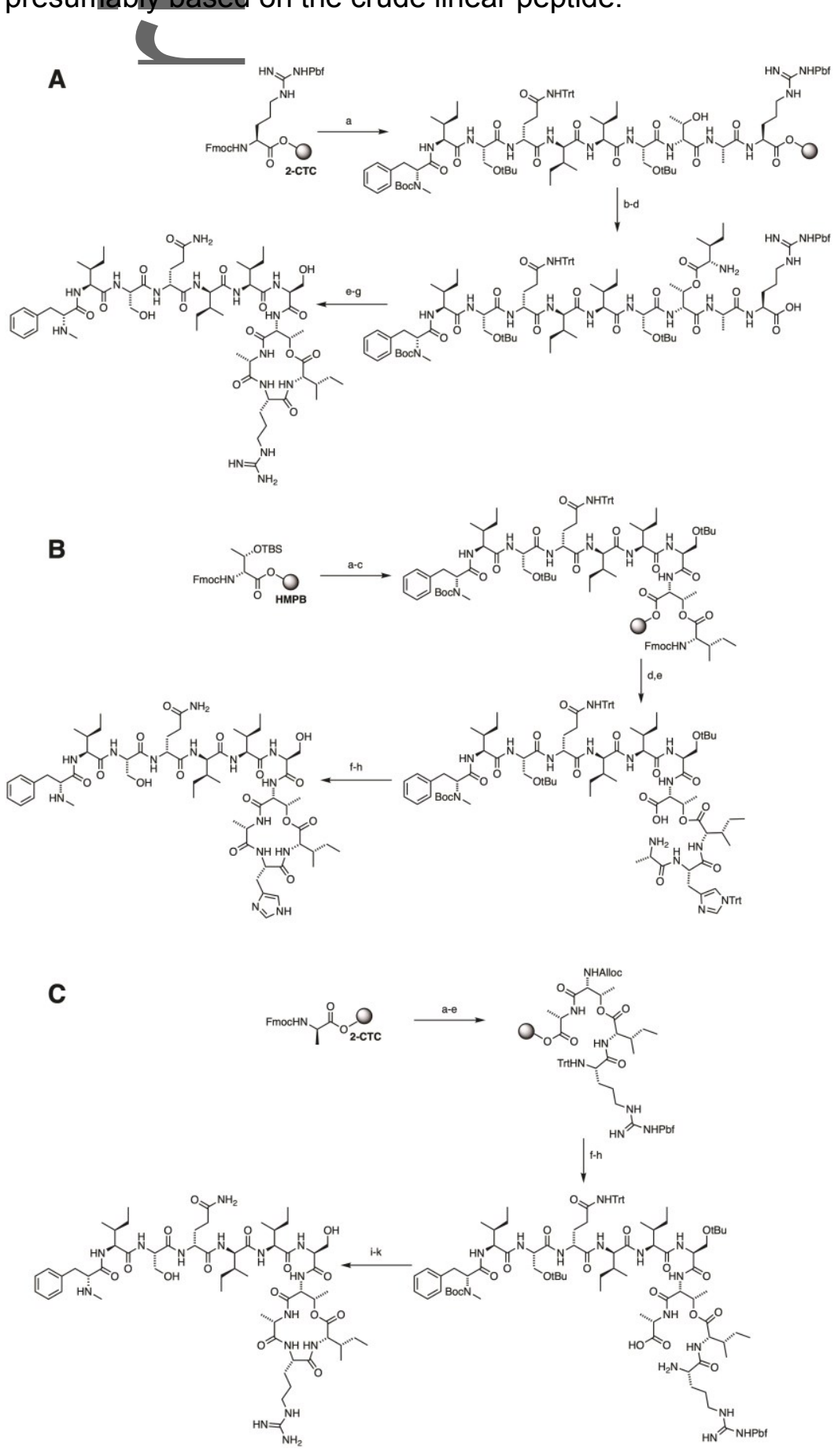

This article is protected by copyright. All rights reserved. 
Figure 6. Convergent synthetic strategies. (A) Li et al.: (a) Boc SPPS; (b) Ozonolysis; (c) $\mathrm{AcOH}$, TFE, and DCM; (d) HATU, HOAt, and Oxyma Pure; (e) TFA; (f) Pd(OH) $2 / \mathrm{C}$ and $\mathrm{H}_{2}$; (g) Pyridine and $\mathrm{AcOH}$; (h) TFA; and (i) RP-HPLC. Cyclization yield = 36.8\%. (B) Rao et al.: (a) Fmoc SPPS; (b) TFE and DCM; (c) Pd(OH) $/ 2$ C and $\mathrm{H}_{2}$; (d) PyAOP, HOAt, and DIEA; (e) 4N HCland dioxane; (f) DEPBT and DIEA; (g) TFA, TIS, and $\mathrm{H}_{2} \mathrm{O}$; and (h) RP-HPLC. Yield $=12 \%$ from the starting material. (C) Synthetic strategy for N-Me analogues. C-terminal macrocycle: (a) Fmoc- L-Ile-OH, DIC, DMAP, and DCM, 2 h; (b) Fmoc SPPS; (c) 1\% TFA, DCM; (d) 3eq. EDC.HCl, 5 eq. DIEA, and DCM, 4 mM; (e) 1\% $\mathrm{H}_{2} \mathrm{O}$, TFA; (f) RP-HPLC; (g) 3 eq. COMU, 5 eq. DIEA, and 1,4-dioxane; (h) $1 \%$ TIPS, $2 \% \mathrm{H}_{2} \mathrm{O}$, and TFA; and (i) RPHPLC.Yields of up to $17 \%$ were reported based on the fragment condensation and global

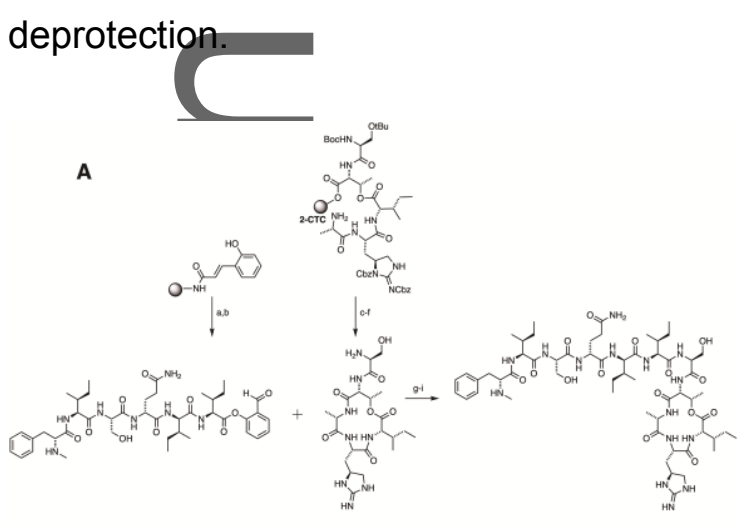

B
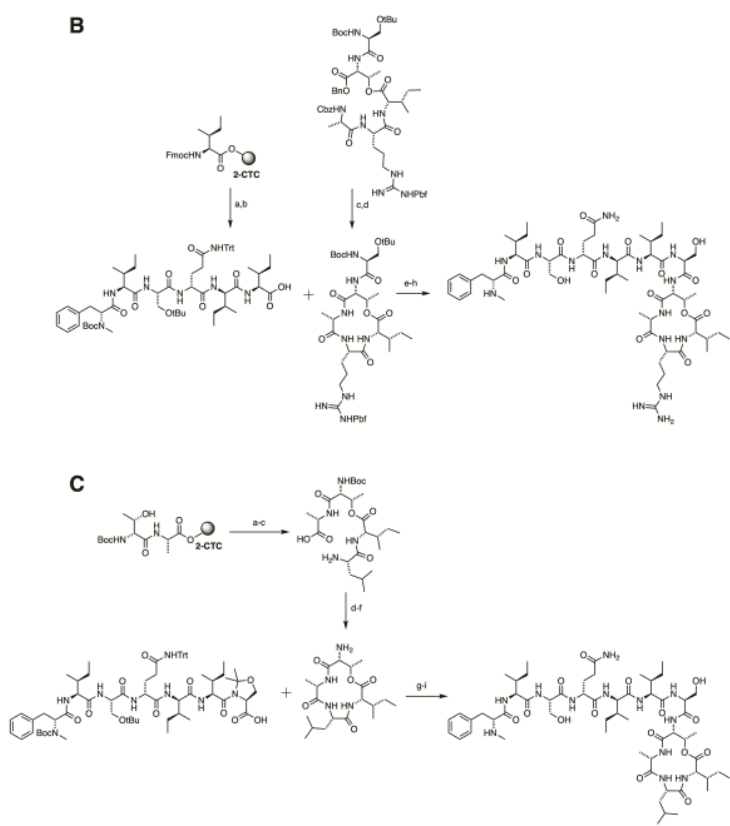

This article is protected by copyright. All rights reserved. 
Figure 7. "On-resin" macrocyclization. (A) Albericio et al.: (a) Alloc-lle-OH, DIC, and DMAP; (b) $\mathrm{Pd}\left(\mathrm{PPh}_{3}\right)_{4}$ and $\mathrm{PhSiH}_{3}$; (c) PyAOP and Oxyma Pure; (d) Fmoc SPPS; (e) TFA, TIS, and $\mathrm{H}_{2} \mathrm{O}$; (f) TEA, TIS, and $\mathrm{H}_{2} \mathrm{O}$; (h) RP-HPLC. Yields not reported. (B) Su et al.: (a) Fmoc SPPS; (b) Fmoc-lle-OH, DCC, and DMAP; (c) Fmoc SPPS; (d) Cu(OAc)2, Pyridine; (e) TFA, TIS, and $\mathrm{H}_{2} \mathrm{O}$; and (f) RP-HPLC. Yield $=18 \%$.
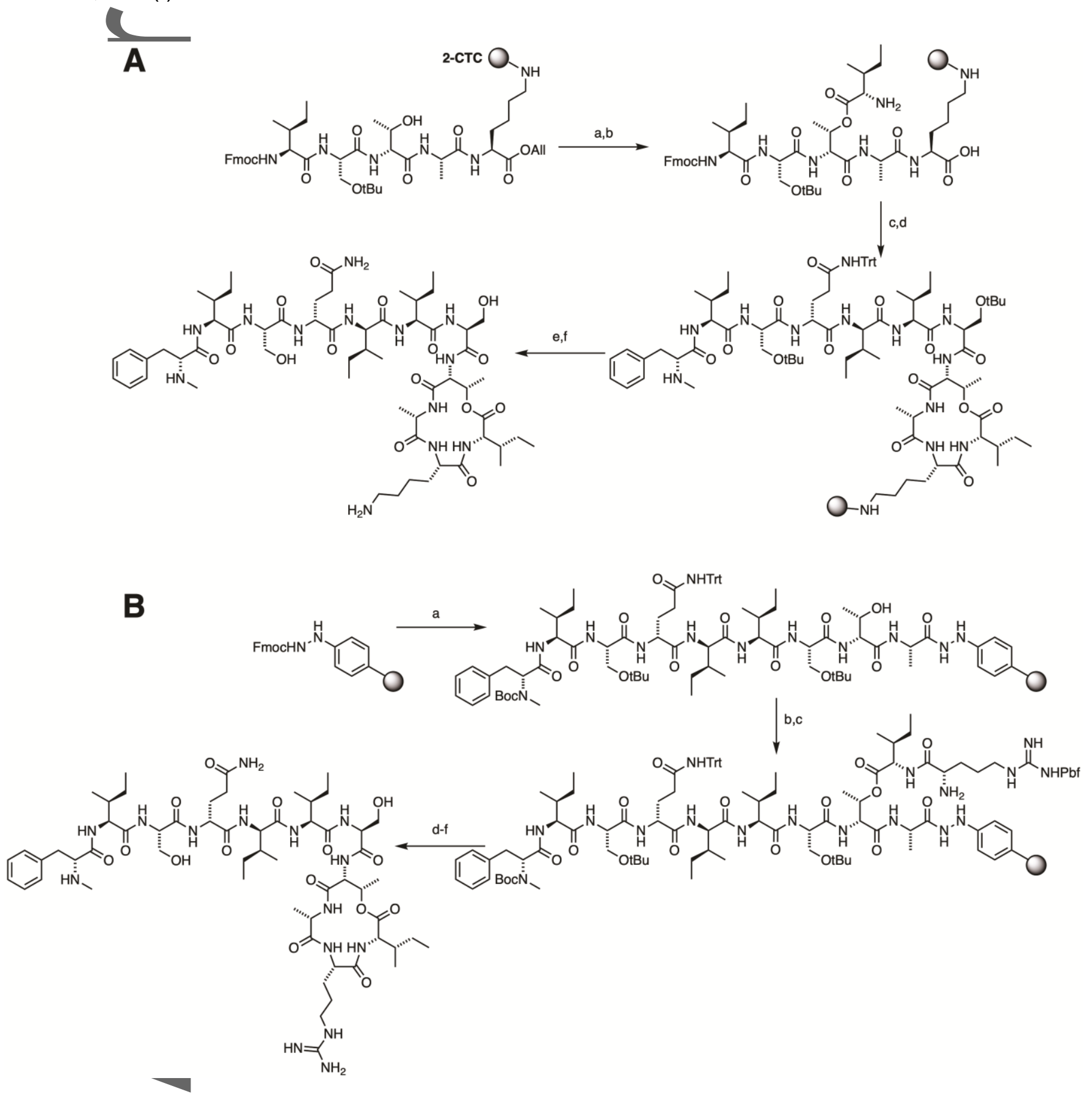

This article is protected by copyright. All rights reserved. 
Figure 8. The teixobactin macrocycle. (A) Scale-up synthesis of the C-terminal macrocycle by Reddy et al.: (a) HATU, DIEA; (b) TBAF; (c) LiOH and $\mathrm{H}_{2} \mathrm{O}$; (d) MNBA, DMAP, Dy(OTf) and DIEA; (e) TFA. Yield = 30-35\%. (B) Simplified cyclic analogues by Jamieson et al:: (a) 1\% TFA in DCM; (b) HATU and DIEA; (c) TFA, EDT, TES, and DCM; (d) Zn(OAc)2, geranyl$\mathrm{Br}$, and TFA; and (e) RP-HPLC. Yields of up to $12 \%$ were reported. (C) Disulfide linked macrocycle by Singh et al:: (a) Fmoc SPPS; (b) TFA, TIS, and $\mathrm{H}_{2} \mathrm{O}$; (c) DMSO, and $\mathrm{H}_{2} \mathrm{O}$; and (d) RP-HPLC. Yields were in the range of 15-25\%.
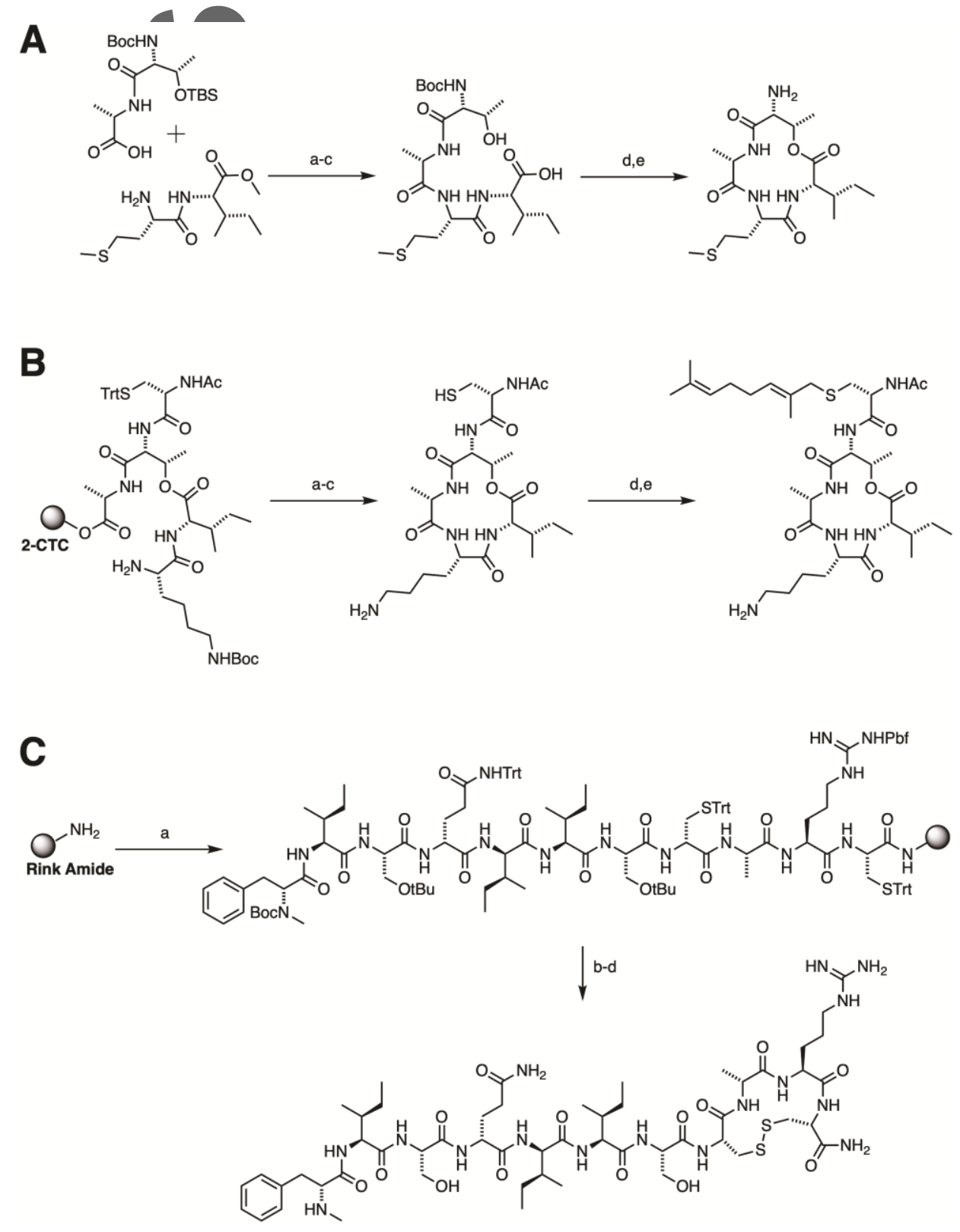

This article is protected by copyright. All rights reserved. 
Figure 9. Alternative approaches. (A) Solution phase synthesis of teixobactin by Gao et al::

(a) $\mathrm{LiOH}$, THF, and $\mathrm{H}_{2} \mathrm{O}$; (b) L-Ser-OMe, PyAOP, DIEA, and DCM; (c) TBSCl, imidazole, and DCM; (d) $\mathrm{Me}_{3} \mathrm{SnOH}$ and PhMe; (e) LiOH, THF, and $\mathrm{H}_{2} \mathrm{O}$; (f) L-lle-OMe, PyAOP, DIEA, and DCM; (g) $\mathrm{PdCl}_{2}, \mathrm{H}_{2}$, and MeOH; (h) PyAOP, DIEA, and DMF; (i) Et2NH, and DCM; (j) PyAOP,DIEA, and DCM; (k) TFA/DCM; (I) HATU, HOAt, NMM, and DMF; (m) Pd(PPh $)_{4}$, $\mathrm{Et}_{2} \mathrm{NH}$, amd DCM; (n) $\mathrm{Me}_{3} \mathrm{SnOH}, \mathrm{PhMe}$; (o) EDCl, HOAt, DIEA, and DMF; (p) $\mathrm{PdCl}_{2}, \mathrm{H}_{2}$, and $\mathrm{MeOH}$; and (q) TFA, TIS, and DCM. (B) Gram-scale synthesis of native teixobactin using a convergent $2+3+6$ approach by Rao et al." (a) Fmoc SPPS; (b) TFE and DCM; (c) DEPBT and DIEA; (d) Pd( $\left(\mathrm{PPh}_{3}\right)_{4}$ and 1,3-dimethylbarbituric acid; (e) HATU, HOAt, and DIEA; (f) $\mathrm{HCl}$; (g) DEPBT and DIEA; (h) $\mathrm{Pd}(\mathrm{OH})_{2} / \mathrm{C}$ and $\mathrm{H}_{2}$; (i) TFA, TIS and $\mathrm{H}_{2} \mathrm{O}$; and (j) RP-HPLC. Yield $=8 \%$. (C) Chemoenzymatic synthesis of teixobactin by Mandalapu et al. (a) FmocSPPS; (b) $2 \%$ TFA in DCM; (c) L-Ile-OMe, HBTU, HOBt, DIEA, and DCM/DMF; (d) TFA, TIS, and $\mathrm{H}_{2} \mathrm{O}$; and (e) thioesterase enzyme and aqueous buffer.

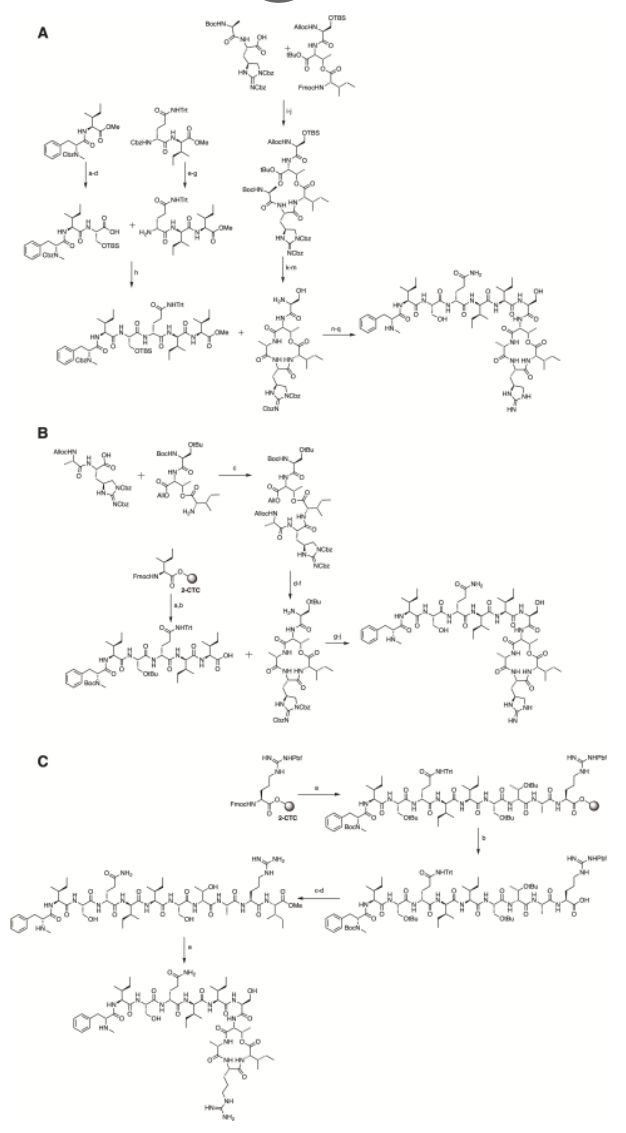

This article is protected by copyright. All rights reserved. 
Figure 10. Summary of modifiable and sensitive residues in teixobactin. Blue: Na-amides thought to be responsible for pyrophosphate binding in lipid II. These are likely to be sensitive to modification. Red: regions usually highly sensitive to modification. Orange: regions that can tolerate some modification. Green: highly modifiable residues. Please note that Singh and coworkers were able to alter residues 2, 5, 6, or 7 and still generate active analogues, provided that other cationic substitutions were also made. ${ }^{49}$

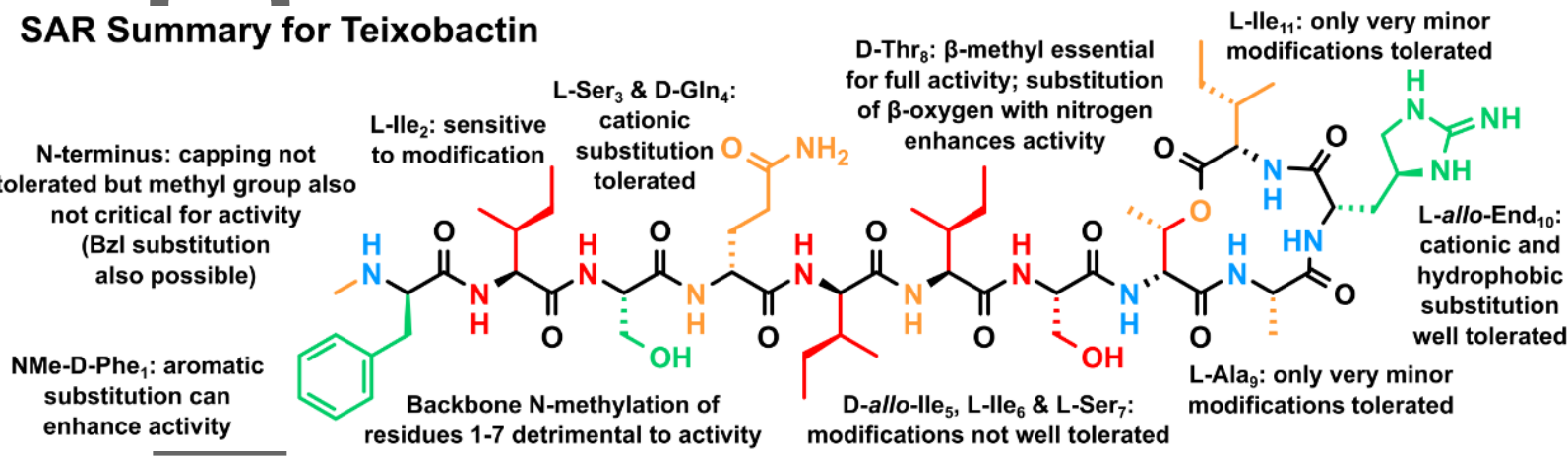

Figure 11. (A) Molecular modeling of possible amyloid-like assemblies of teixobactin. (B)

Model of the interaction of teixobactin with lipid II.
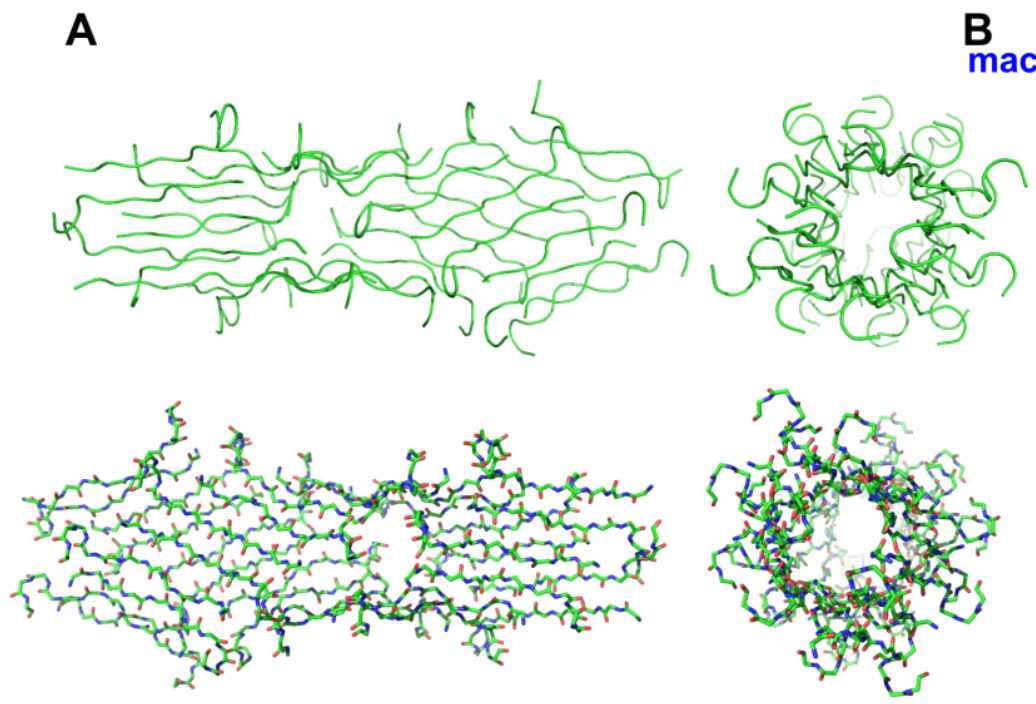

macrocycle 'cage'

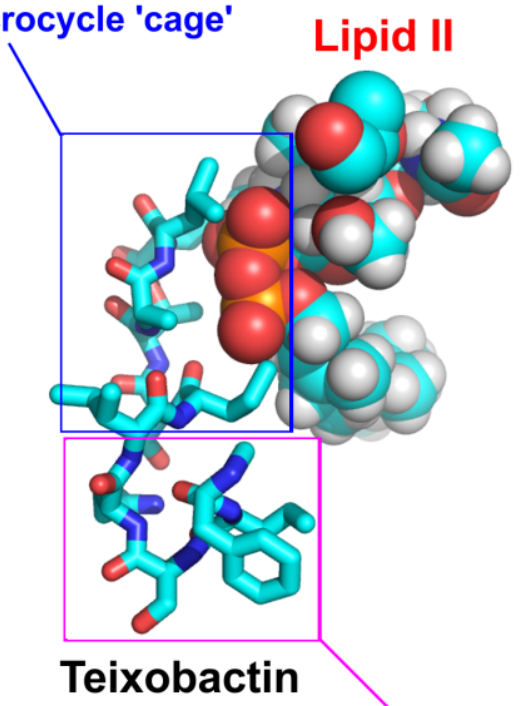

$N$-terminal 'tail'

This article is protected by copyright. All rights reserved. 


\section{References}

1. WHO. 2014. Antimicrobial resistance: global report on surveillance 2014.

2. O'Neill, J. 2016. Tackling drug-resistant infections globally: final report and recommendations. The Review on Antimicrobial Resistance.

3. Liu, Y. Y., Wang, Y., Walsh, T. R., et al. 2016. Emergence of plasmid-mediated colistin resistance mechanism MCR-1 in animals and human beings in China: a microbiological and molecular biological study. The Lancet. Infectious diseases. 16, 2: 161-8.

4. Walsh, T. R. Wu, Y. 2016. China bans colistin as a feed additive for animals. The Lancet. Infectious diseases. 16, 10: 1102-1103.

5. Venter, H., Henningsen, M. L., Begg, S. L. 2017. Antimicrobial resistance in healthcare, agriculture and the environment: the biochemistry behind the headlines. Essays Biochem. 61, 1: 1-1

6. Fiore, D. C. Fettic, L. P., Wright, S. D., et al. 2017. Antibiotic overprescribing: still a major concern. The Journal of family practice. $66,12: 730-736$.

7. Talbot, G. H., Bradley, J., Edwards, J. E., et al. 2006. Bad bugs need drugs: an update on the development pipeline from the Antimicrobial Availability Task Force of the IDSA. Clin. Infect. Dis 42, 5: 657-68.

8. Jonas, O. B., Irwin, A., Berthe, F. C. J., et al. 2017. Drug-resistant infections : a threat to our economic future, 2017. HNP/Agriculture Global Antimicrobial Resistance Initiative.

Washington, D.C.: World Bank Group.

9. Lewis, K. 2017. Platforms for antibiotic discovery. Nat. Rev. Drug Discov. 12, 5: 371-87. 10. Ling, L. L., Schneider, T., Peoples, A. J., et al. 2015. A new antibiotic kills pathogens without detectable resistance. Nature. 517, 7535: 455-9.

11. Pham, V. H, Kim, J. 2012. Cultivation of unculturable soil bacteria. Trends Biotechnol. 30, 9: 475-84. 
12. Nichols, D., Cahoon, N., Trakhtenberg, E. M., et al. 2010. Use of ichip for highthroughput in situ cultivation of "uncultivable" microbial species. Appl. Environ. Microbiol. 76, 8: 2445-50.

13. Fiers, W. D., Craighead, M., Singh, I. 2017. Teixobactin and its analogues: A new hope in antibiotic discovery. ACS Infect. Dis. 3, 10: 688-690.

14. (a) Alrabiah, K., Al Alola, S., Al Banyan, E., et al. 2016. Characteristics and risk factors of hospital acquired - methicillin-resistant Staphylococcus aureus (HA-MRSA) infection of pediatric patients in a tertiary care hospital in Riyadh, Saudi Arabia. Int. J. Pediatr. Adolesc. Med. 3, 2: 71-77. (b) DeLeo, F. R., Otto, M., Kreiswirth, B. N., et al. 2010. Communityassociated meticillin-resistant staphylococcus aureus. Lancet (London, England). 375, 9725: 1557-68.

15. (a) Jakab, E., Zbinden, R., Gubler, J., et al. 1996. Severe infections caused by Propionibacterium acnes: an underestimated pathogen in late postoperative infections. Yale J. Biol. Med. 69, 6: 477-82. (b) Achermann, Y., Goldstein, E. J., Coenye, T., et al. 2014. Propionibacterium acnes: from commensal to opportunistic biofilm-associated implant pathogen. Clin. Microbiol. Rev. 27, 3: 419-40.

16. von Nussbaum, F., Sussmuth, R. D. 2015. Multiple attack on bacteria by the new antibiotic teixobactin. Angew. Chem. Int. Ed. Engl. 54, 23: 6684-6.

17. Homma, T., Nuxoll, A., Gandt, A. B., et al. 2016. Dual targeting of cell wall precursors by teixobactin leads to cell lysis. Antimicrob. Agents Ch. 60, 11: 6510-6517.

18. de Kruijff, B., van Dam, V., Breukink, E. 2008. Lipid II: a central component in bacterial cell wall synthesis and a target for antibiotics. Prostaglandins Leukotr. Ess. 79, 3-5: 117-21. 19. Sewell, E. W.,Brown, E. D. 2014. Taking aim at wall teichoic acid synthesis: new biology and new leads for antibiotics. J. Antibiot. (Tokyo). 67, 1: 43-51.

20. Koch, A.L. 2003. Bacterial wall as target for attack: past, present, and future research. Clin. Microbiol. Rev. 16, 4: 673-87. 
21. (a) Ng, V., Chan, W. C. 2016. New found hope for antibiotic discovery: lipid II inhibitors. Chemistry. 22, 36: 12606-16. (b) Oppedijk, S. F., Martin, N. I., Breukink, E. 2016. Hit 'em where it hurts: the growing and structurally diverse family of peptides that target lipid-II. Biochim. Biophys. Acta. 1858, 5: 947-57.

22. Breukink, E., de Kruijff, B. 2006. Lipid II as a target for antibiotics. Nat. Rev. Drug Discov. 5, 4: 321-32.

23. Hamburger, J. B., Hoertz, A. J., Lee, A. et al. 2009. A crystal structure of a dimer of the antibiotic ramoplanin illustrates membrane positioning and a potential lipid II docking interface. Proc. Natl Acad. Sci. USA. 106, 33: 13759-64.

24. Brown, S., Santa Maria Jr, J. P., Walker, S. 2013. Wall teichoic acids of Gram-positive bacteria. Annu. Rev. Microbiol. 67: 313-36.

25. Soldo,B., Lazarevic, V., Karamata, D. 2002. TagO is involved in the synthesis of all anionic cell-wall polymers in Bacillus subtilis 168. Microbiology. 148, Pt 7: 2079-87. 26. (a) Guo, C., Mandalapu, D., Ji, X., et al. 2018. Chemistry and biology of teixobactin. Chem. Eur. J. 24, 21: 5406-5422. (b) Abdel Monaim, S. A. H., Jad, Y. E., El-Faham, A., et al. 2018. Teixobactin as a scaffold for unlimited new antimicrobial peptides: SAR study. Bioorg. Med. Chem. 26, 10: 2788-2796.

27. Atkinson, D. J., Naysmith, B. J., Furkert, et al. 2016. Enduracididine, a rare amino acid component of peptide antibiotics: natural products and synthesis. Beilstein J. Org. Chem. 12: 2325-2342

28. Jad, Y. E., Acosta, G. A., Naicker, T., et al. 2015. Synthesis and biological evaluation of a teixobactin analogue. Org. Lett. 17, 24: 6182-6185.

29. (a) Giltrap, A. M., Dowman, L. J., Nagalingam, et al. 2016. Total synthesis of teixobactin. Org. Lett. 18, 11:2788-2791. (b) Jin, K., Sam, I. H., Po, K. H., et al. 2016. Total synthesis of teixobactin. Nat. Commun. 7: 12394. 
30. Zong, Y., Fang, F., Meyer, K. J., et al. 2019. Gram-scale total synthesis of teixobactin promoting binding mode study and discovery of more potent antibiotics. Nat. Commun. 10, 1: 3268

31. Yang, H., Chen, K. H., Nowick, J. S. 2016. Elucidation of the teixobactin pharmacophore. ACS Chem. Biol. 11, 7: 1823-1826.

32. Ng, V., Kuehne, S. A., Chan, W. C. 2018. Rational design and synthesis of modified teixobactin analogues: in vitro antibacterial activity against Staphylococcus aureus, Propionibacterium acnes and Pseudomonas aeruginosa. Chem. Eur. J. 24, 36: 9136-9147. 33. Schumacher, C. E., Harris, P. W. R., Ding, X.-B., et al. 2017. Synthesis and biological evaluation of novel teixobactin analogues. Org. Biomol. Chem. 15, 41: 8755-8760.

34. Parmar, A., lyer, A., Vincent, C. S., et al. 2016. Efficient total syntheses and biological activities of two teixobactin analogues. Chem. Commun. 52, 36: 6060-6063.

35. Zhang, Y., Xu, C., Lam, H. Y., et al. 2013. Protein chemical synthesis by serine and threonine ligation. PNAS. 110, 17: 6657-6662.

36. Zong, Y., Sun, X., Gao, H., et al. 2018. Developing equipotent teixobactin analogues against drug-resistant bacteria and discovering a hydrophobic interaction between lipid II and teixobactin. J. Med. Chem. 61, 8: 3409-3421.

37. Velkov, T., Swarbrick, J. D., Hussein, M. H., et al. 2019. The impact of backbone Nmethylation on the structure-activity relationship of Leu10-teixobactin. J. Pept. Sci. 25, 9: e3206.

38. Abdel Monaim, S. A. H., Ramchuran, E. J., El-Faham, A., et al. 2017. Converting teixobactin into a cationic antimicrobial peptide (AMP). J. Med. Chem. 60, 17: 7476-7482. 39. Wu, C., Pan, Z., Yao, G., et al. 2017. Synthesis and structure-activity relationship studies of teixobactin analogues. RSC Adv. 7, 4: 1923-1926.

40. Dhara, S., Gunjal, V. B., Handore, et al. 2016. Solution-phase synthesis of the macrocyclic core of teixobactin. Eur. J. Org. Chem. 2016, 25: 4289-4293. 
41. Girt, G. C., Mahindra, A., Al Jabri, Z. J. H., et al. 2018. Lipopeptidomimetics derived from teixobactin have potent antibacterial activity against Staphylococcus aureus. Chem. Commun. 2018, 54 (22), 2767-2770.

42. Malkawi, R., Jyer, A., Parmar, A., et al. 2018. Cysteines and disulfide-bridged macrocyclic mimics of teixobactin analogues and their antibacterial activity evaluation against methicillin-resistant Staphylococcus aureus (MRSA). Pharmaceutics. 10, 4: 183. 43. Gao, B., Chen, S., Hou, Y. N., et al. 2019. Solution-phase total synthesis of teixobactin. Org. Biomol Chem. 17, 5: 1141-1153.

44. Mandalapu,D., Ji, X., Chen, J., et al. 2018. Thioesterase-mediated synthesis of teixobactin analogues: mechanism and substrate specificity. J. Org. Chem. 83, 13: 72717275 .

45. Abdel Monaim, S. H., Jad, Y. E., Acosta, G. A., et al. 2016. Re-evaluation of the Nterminal substitution and the D-residues of teixobactin. RSC Adv. 6, 77: 73827-73829. 46. Yang, H., Wierzbicki, M., Du Bois, D. R., et al. 2018. X-ray crystallographic structure of a teixobactin derivative reveals amyloid-like assembly. J. Am. Chem. Soc. 140, 43: 1402814032

47. Wen, P. C., Vanegas, J. M., Rempe, S. B., et al. 2018. Probing key elements of teixobactin-lipid II interactions in membranes. Chem. Sci. 9, 34: 6997-7008. 48. (a) Jin, K., Po, K. H. L., Wang, S., et al. 2017. Synthesis and structure-activity relationship of teixobactin analogues via convergent Ser ligation. Bioorg. Med. Chem. 25, 18: 4990-4995. (b) Chen, K. H., Le, S. P., Han, X., et al. 2017. Alanine scan reveals modifiable residues in teixobactin. Chem. Commun. 53, 82: 11357-11359.

49. Parmar, A., Lakshminarayanan, R., lyer, A., et al. 2018. Design and syntheses of highly potent teixobactin analogues against Staphylococcus aureus, methicillin-resistant Staphylococcus aureus (MRSA), and vancomycin-resistant Enterococci (VRE) in vitro and in vivo. J. Med. Chem. 61, 5: 2009-2017. 
50. Abdel Monaim, S. A. H., Noki, S., Ramchuran, E. J., et al. 2017. Investigation of the Nterminus amino function of Arg10-teixobactin. Molecules. 22, 10: 1632.

51. Parmar, A., Prior, S. H., lyer, A., et al. 2017. Defining the molecular structure of teixobactin analogues and understanding their role in antibacterial activities. Chem. Commun. 53, 12:-2016-2019.

52. Parmar, A., lyer, A., Prior, S. H., et al. 2017. Teixobactin analogues reveal enduracididine to be non-essential for highly potent antibacterial activity and lipid II binding. Chem. Sci. 8, 12: 8183-8192.

53. Abdel Monaim, S. A. H., Jad, Y. E., Ramchuran, E. J., et al. 2016. Lysine scanning of Arg10-teixobactin: deciphering the role of hydrophobic and hydrophilic residues. ACS Omega. 1,6: 1262-1265.

54. Yang, H., Du Bois, D. R., Ziller, J. W., et al. 2017. X-ray crystallographic structure of a teixobactin analogue reveals key interactions of the teixobactin pharmacophore. Chem. Commun. 53, 18: 2772-2775.

55. Craig, W., Chen, J., Richardson, D., et al. 2015. A highly stereoselective and scalable synthesis of L-allo-enduracididine. Org. Lett. 17, 18: 4620-4623.

56. Parmar, A., lyer, A., Lloyd, D. G., et al. 2017. Syntheses of potent teixobactin analogues against methicillin-resistant Staphylococcus aureus (MRSA) through the replacement of Lallo-enduracididine with its isosteres. Chem. Commun. 53, 55: 7788-7791.

57. Jin, K., Po, K.H. L., Kong, W. Y., et al. 2018. Synthesis and antibacterial studies of teixobactin analogues with non-isostere substitution of enduracididine. Bioorg. Med. Chem. 26, 5: 1062-1068.

58. Öster, C., Walkowiak, G. P., Hughes, D. E., et al. 2018. Structural studies suggest aggregation as one of the modes of action for teixobactin. Chem. Sci. 9, 47: 8850-8859. 59. (a) Baver, R., Dicks, L. M. 2005. Mode of action of lipid II-targeting lantibiotics. Int. J. Food Microbiol. 101, 2: 201-16. (b) Hsu, S. T., Breukink, E., Tischenko, et al. 2004. The 
nisin-lipid II complex reveals a pyrophosphate cage that provides a blueprint for novel antibiotics. Nat. Struct. Mol. Biol. 11, 10: 963-7.

60. Liu, Y., Liu, Y., Chan-Park, M. B., et al. 2017. Binding modes of teixobactin to lipid II: molecular dynamics study. Sci. Rep. 7, 1: 17197.

61. McCarthy, M.W. 2019. Teixobactin: a novel anti-infective agent. Expert Rev. Anti. Infect. Ther. 17, 1: 1-3.

62. Ramchuran, E. J., Somboro, A. M., Abdel Monaim, S. A. H., et al. 2018. In vitro antibacterial activity of teixobactin derivatives on clinically relevant bacterial isolates.

Front.Microbiol.9: 1535.

63. Chiorean, S., Antwi, I., Carney, D. W., et al. 2019. Dissecting the binding interactions of teixobactin with the bacterial cell wall precursor lipid II. ChemBioChem. DOI:

10.1002/cbic.201900504.

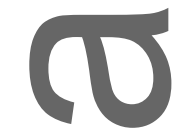

Table 1. Summary of the effect of substitution on antimicrobial activity of teixobactin by residue

\begin{tabular}{|c|c|c|c|c|c|c|c|c|c|c|c|c|}
\hline \multirow[b]{2}{*}{ Modification } & \multicolumn{12}{|c|}{ RESIDUES } \\
\hline & $\begin{array}{c}\mathrm{N}- \\
\text { terminu } \\
\mathrm{S}\end{array}$ & $\begin{array}{c}\text { NMe-D- } \\
\text { Phe }_{1}\end{array}$ & L-Ile 2 & $\mathrm{~L}_{-} \mathrm{Ser}_{3}$ & $\begin{array}{c}\mathrm{D}- \\
\mathrm{G} \ln _{4}\end{array}$ & $\begin{array}{c}\text { D-allo- } \\
\mathrm{lle}_{5}\end{array}$ & L-Ile 6 & L-Ser 7 & D-Thr & L-Ala 9 & $\begin{array}{l}\text { L-allo- } \\
\text { End }_{10}\end{array}$ & L-IIe 11 \\
\hline Minor & $x$ & $\checkmark$ & $\mathrm{O}$ & $\checkmark$ & $\checkmark$ & $\mathrm{O}$ & $x$ & 0 & $\checkmark$ & $\mathrm{O}$ & $\checkmark$ & $\mathrm{O}$ \\
\hline Alanine & - & $x$ & $x$ & $\checkmark$ & $\checkmark$ & $x$ & $x$ & $\mathrm{O}$ & $x$ & - & $\checkmark$ & $\mathrm{O}$ \\
\hline Cationic & - & $x$ & $x$ & $\checkmark$ & $\checkmark$ & $x$ & $x$ & 0 & - & 0 & $\checkmark$ & $x$ \\
\hline Anionic & - & $?$ & $?$ & $x$ & ? & $?$ & $?$ & $?$ & - & $?$ & $x$ & $?$ \\
\hline Hydrophobic & $x$ & $?$ & - & $?$ & $?$ & - & - & $?$ & - & $?$ & $\checkmark$ & - \\
\hline Aromatic & $x$ & $\checkmark$ & $x$ & $?$ & $?$ & $?$ & $x$ & $?$ & - & $x$ & $\mathrm{O}$ & $?$ \\
\hline$N^{\alpha}$-methylation & 0 & - & $x$ & $x$ & 0 & $x$ & 0 & $x$ & ? & ? & ? & ? \\
\hline $\begin{array}{l}\text { Stereochemis } \\
\text { switch }\end{array}$ & - & $x$ & $x$ & $?$ & $x$ & $x$ & $?$ & $?$ & $x$ & $x$ & $\mathrm{O}$ & $\mathrm{O}$ \\
\hline
\end{tabular}

Note: $\sqrt{ }$, amenable to modification; $O$, sometimes amenable to modification or results in a minor loss of activity; $X$, not amenable to modification; ?, has not been evaluated; -, not applicable. Please note that this table is a rough guide only. 

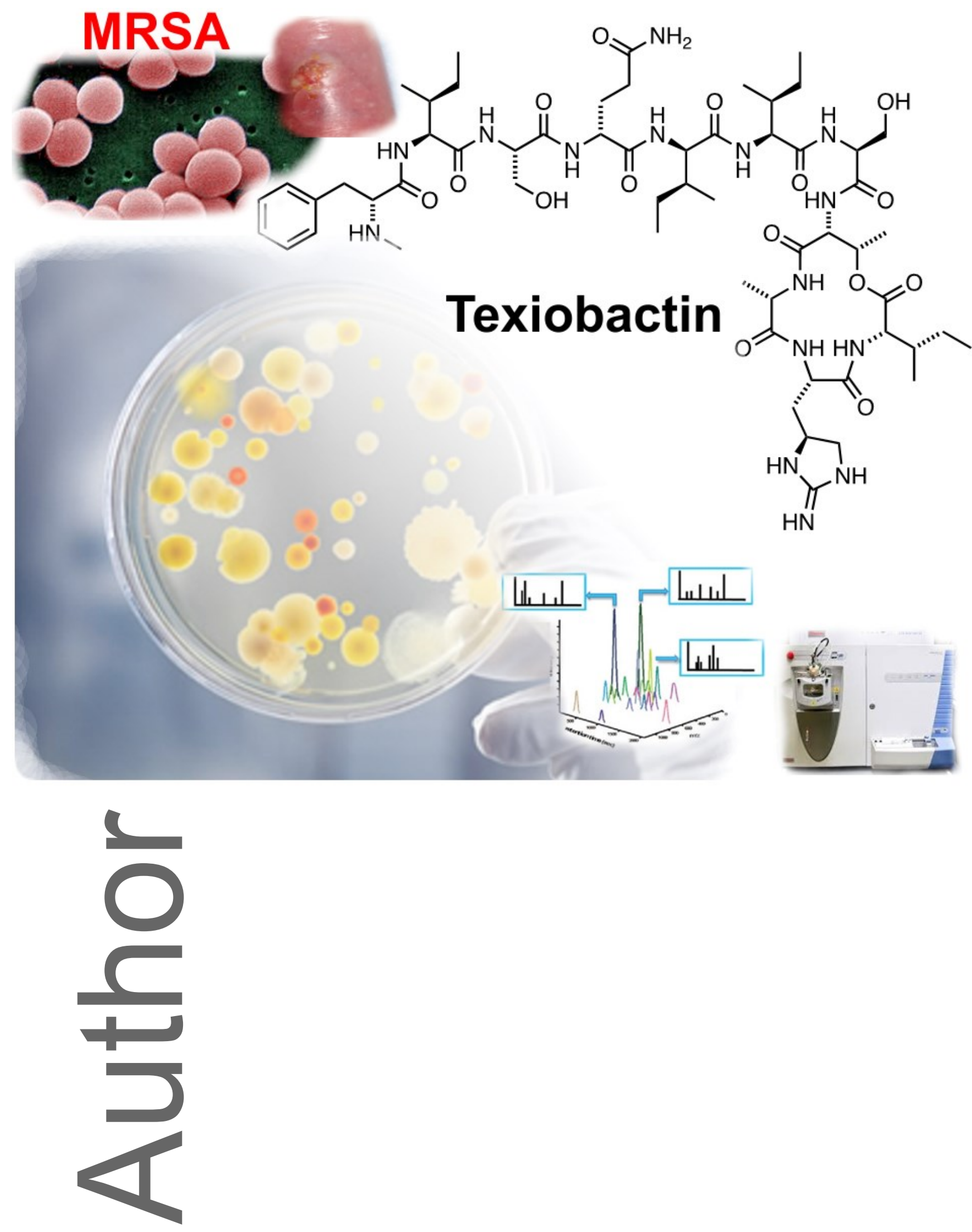

This article is protected by copyright. All rights reserved. 


\section{University Library}

\section{- M M N E R VA A gateway to Melbourne's research publications}

Minerva Access is the Institutional Repository of The University of Melbourne

\section{Author/s:}

Karas, JA;Chen, F;Schneider-Futschik, EK;Kang, Z;Hussein, M;Swarbrick, J;Hoyer,

D;Giltrap, AM;Payne, RJ;Li, J;Velkov, T

Title:

Synthesis and structure-activity relationships of teixobactin

Date:

2019-12-02

\section{Citation:}

Karas, J. A., Chen, F., Schneider-Futschik, E. K., Kang, Z., Hussein, M., Swarbrick, J., Hoyer, D., Giltrap, A. M., Payne, R. J., Li, J. \& Velkov, T. (2019). Synthesis and structure-activity relationships of teixobactin. ANNALS OF THE NEW YORK ACADEMY OF SCIENCES, 1459 (1), pp.86-105. https://doi.org/10.1111/nyas. 14282.

Persistent Link:

http://hdl.handle.net/11343/286706 